\title{
1 The longitudinal dynamics and natural history of clonal haematopoiesis
}

2 Margarete A. Fabre ${ }^{1,2,3 \star}$, José Guilherme de Almeida ${ }^{4 *}$, Edoardo Fiorillo ${ }^{5}$, Emily Mitchell ${ }^{1,2,3}$, 3 Aristi Damaskou, ${ }^{2,3}$, Justyna Rak ${ }^{2,3}$, Valeria Orrù ${ }^{5}$, Michele Marongiu ${ }^{5}$, MS Vijayabaskar ${ }^{2}$, 4 Joanna Baxter ${ }^{6}$, Claire Hardy ${ }^{1}$, Federico Abascal ${ }^{1}$, Michael Spencer Chapman ${ }^{1,2,3}$, Nicholas 5 Williams $^{1,2}$, Jyoti Nangalia ${ }^{1,2,3}$, Iñigo Martincorena ${ }^{1}$, Peter J. Campbell ${ }^{1,2}$, Eoin F. McKinney ${ }^{7}$, 6 Francesco Cucca ${ }^{5,8}$, Moritz Gerstung ${ }^{4 \dagger}$, George S. Vassiliou ${ }^{1,2,3 \dagger}$.

1. Wellcome Sanger Institute, Wellcome Genome Campus, Cambridge, CB10 1SD, UK

2. Wellcome-MRC Cambridge Stem Cell Institute, University of Cambridge, Cambridge, CB2 OXY, UK

3. Department of Haematology, University of Cambridge, Cambridge, CB2 OXY, UK.

4. European Bioinformatics Institute, Wellcome Genome Campus, Cambridge, CB10 1SD, UK

5. Istituto di Ricerca Genetica e Biomedica, Consiglio Nazionale delle Ricerche, Lanusei, Italy

6. Cambridge Blood and Stem Cell Biobank, Department of Haematology, University of Cambridge, Cambridge, CB2 OAW, UK

7. Cambridge Institute of Therapeutic Immunology \& Infectious Disease, University of Cambridge, Cambridge, CB2 OAW, UK

8. Dipartimento di Scienze Biomediche, Università degli Studi di Sassari, Sassari, Italy

* These authors contributed equally to the work 


\section{Summary}

21 Human cells acquire somatic mutations throughout life, some of which can drive clonal 22 expansion. Such expansions are frequent in the haematopoietic system of healthy individuals 23 and have been termed clonal haematopoiesis $(\mathrm{CH})$. While $\mathrm{CH}$ predisposes to myeloid 24 neoplasia and other diseases, we have limited understanding of how and when $\mathrm{CH}$ develops, what factors govern its behaviour, how it interacts with ageing and how these variables relate to malignant progression. Here, we track $697 \mathrm{CH}$ clones from 385 individuals aged 55 or older over a median of 13 years. We find that $92.4 \%$ of clones expanded at a stable exponential rate over the study period, with different mutations driving substantially different growth rates, ranging from 5\% (DNMT3A, TP53) to over 50\%/yr (SRSF2-P95H). Growth rates of clones with the same mutation differed by approximately $+/-5 \% / y r$, proportionately impacting "slow" drivers more substantially. By combining our time-series data with phylogenetic analysis of 1,731 whole genome-sequenced haematopoietic colonies from 7 older individuals, we reveal distinct patterns of lifelong clonal behaviour. DNMT3A-mutant clones preferentially expanded early in life and displayed slower growth in old age, in the context of an increasingly competitive oligoclonal landscape. By contrast, splicing gene mutations only drove expansion later in life, while growth of TET2-mutant clones showed minimal age-dependency. Finally, we show that mutations driving faster clonal growth carry a higher risk of malignant progression. Our findings characterise the lifelong natural history of $\mathrm{CH}$ and give fundamental insights into the interactions between somatic mutation, ageing and clonal selection. 


\section{Introduction}

42 Human haematopoiesis produces hundreds of billions of specialized blood cells every day, 43 through a hierarchy of progressively more differentiated and numerous cells originating from 44 a pool of long-lived haematopoietic stem cells (HSCs). Haematopoiesis remains highly 45 efficient for decades, but is inevitably challenged by the phthisic effects of ageing ${ }^{1-3}$ and the 46 inexorable acquisition of somatic DNA mutations ${ }^{4}$. Mutations that augment HSC "fitness" can 47 drive clonal expansion of a mutant HSC and its progeny, a phenomenon known as clonal 48 haematopoiesis $(\mathrm{CH})^{5-8}$. $\mathrm{CH}$ becomes ubiquitous with advancing age and is associated with 49 an increased risk of myeloid leukaemias and some non-haematological diseases ${ }^{5-7,9-11}$.

50 The observation that $\mathrm{CH}$-associated mutations affect a very restricted set of genes that are 51 also frequently mutated in leukaemia - most commonly those involved in epigenetic 52 regulation (DNMT3A, TET2 and $A S X L 1$ ), splicing (SF3B1 and SRSF2) and apoptosis (TP53 53 and $P P M 1 D)^{5-8}$ - implies that these mutations inherently confer fitness to HSCs. In fact, recent 54 evolutionary models propose that each specific mutation carries a fixed fitness advantage, 55 and that this explains the relative proportions and clonal sizes of $\mathrm{CH}$ driven by different driver mutations $^{12}$. However, several observations suggest that non-mutation factors are also influential. For example, a handful of $\mathrm{CH}$ cases studied at two time-points propose that the clones driven by the same or very similar mutations can behave differently between individuals ${ }^{11,13}$. Also, the relative prevalence of different $\mathrm{CH}$-driver gene mutations changes significantly depending on context; for example, in aplastic anaemia $\mathrm{CH}$ is commonly driven by mutations that enhance immune evasion ${ }^{14-17}$, whereas genotoxic stress favours clones with mutations in DNA damage genes ${ }^{18-20}$. Furthermore, factors like inflammation ${ }^{21}$ and heritable genetic variation ${ }^{22-24}$ can affect $\mathrm{CH}$ emergence.

64 A major limitation to our understanding of the determinants of $\mathrm{CH}$ behaviour/fate to date has 65 been its reliance on cross-sectional studies capturing $\mathrm{CH}$ at single time-points. Here, by tracking blood cell clones over long periods of time in a large cohort, and by reconstructing haematopoietic phylogenies, we uncover the lifelong dynamics and natural history of $\mathrm{CH}$.

\section{Results}

\section{The age-dependent mutational landscape of $\mathrm{CH}$}

We analysed 1,593 blood DNA samples from 385 adults aged 54-93 years at the time of entry into the SardiNIA longitudinal study ${ }^{25}$. The participants, who had no history of haematological malignancy, were sampled up to 5 times (median 4) over 3.2-16 years (median 12.9 years) (Figure 1a, Extended Data Figure 1a-c). We performed deep targeted sequencing (mean $1,065 x$ ) of 56 genes associated with $\mathrm{CH}$ and haematological malignancy (Supplementary 75 Table 1) and identified somatic mutations in 52 genes (Supplementary Table 2). Using the dNdScv algorithm, an implementation of dN/dS that corrects for trinucleotide mutation rates, sequence composition, and variable mutation rates across genes, we identified positive selection of missense and/or truncating variants in 17 of these genes (dN/dS ratio>1 with $\mathrm{q}<0.1$ ) (Supplementary Table 3, Extended Data Figs. 2,3) ${ }^{26}$. We focussed on these genes for

80 further analysis. 


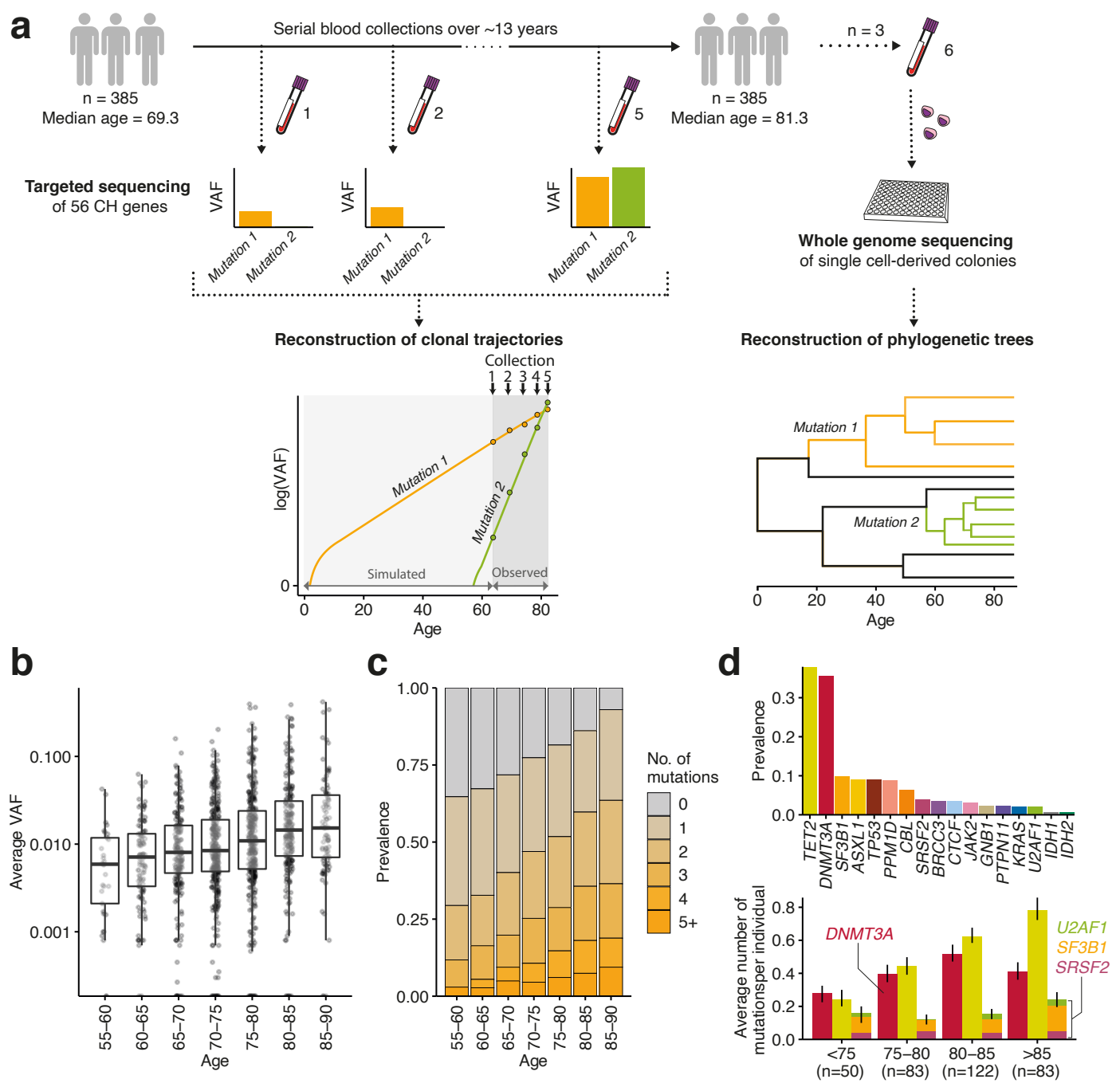

Fig. 1: Experimental workflow and $\mathrm{CH}$ mutation characteristics. a, Study outline: 1,593 blood DNA samples were obtained from 385 elderly individuals sampled 2-5 times (median 4) over 3.2-16 years (median 12.9) and sequenced for mutations in $56 \mathrm{CH}$ genes. Measured variant allele fractions (VAFs) were used to (i) fit observed clonal trajectories, and (ii) extrapolate the clonal dynamics prior to the period of observation. Additional blood samples from 3 selected individuals were used to generate 288 (3x96) whole-genome sequenced single cellderived colonies for phylogeny reconstructions. b, Age distribution of average VAF per individual. c, Age-stratified prevalence of the number of mutations per individual. d, Prevalence of mutations in driver genes: upper panel shows absolute prevalence in the cohort; lower panel shows average number of mutations per individual in DNMT3A, TET2 and splicing genes (SF3B1, SRSF2, U2AF1) at different ages.

At least one somatic non-synonymous mutation was identified in 305 of 385 individuals (79.2\%), with $\mathrm{CH}$ prevalence, average clone size and number of mutations per individual increasing with advancing age, and $\mathrm{CH}$ identified in $>90 \%$ of those aged 85 years or older (Fig. 1b,c). Mutations were most common in epigenetic regulator genes TET2 and DNMT3A, and also frequent in ASXL1, TP53, PPM1D and spliceosome genes (Fig. 1d, upper panel). Interestingly, in this elderly cohort, advancing age impacted the prevalence of different driver mutations in a gene-dependent manner (Fig. 1d, lower panel). In particular, the prevalence of DNMT3A mutations showed no significant relationship with age overall $(p=0.12$, binomial regression of prevalence vs age, controlling for sex), whilst TET2 mutations showed a 
consistent rise with age, averaging at $6.8 \% / \mathrm{yr}(\mathrm{p}=0.00037)$, as did mutations in splicing genes (U2AF1, SRSF2 and SF3B1), whose prevalence increased by $5.4 \% / \mathrm{yr}(\mathrm{p}=0.025)$.

\section{Most clones expand steadily during older age}

105 To investigate clonal behaviour over time, we used serial Variant Allele Fraction (VAF; the 106 fraction of sequencing reads reporting a mutation) measurements as a surrogate for clone 107 size, and fitted a saturating (logistic) exponential curve with a constant growth rate over time 108 to each clonal trajectory. Such logistic growth behaviour is supported by simulations of 109 evolutionary dynamics using Wright-Fisher models with constant fitness (Extended Data Fig. $1104 a-b)^{27}$. Remarkably, by assessing the fit between serial VAF measurements and the 111 trajectories inferred by our model, we find that the great majority of clones (92.4\%) expanded 112 at a constant exponential rate over the study period (Fig. 2a,b, Extended Data Fig. 4c). The 113 predominance of fixed-rate growth was particularly striking for genes like DNMT3A and TET2, 114 for which $99 \%$ and $94.3 \%$ of clones, respectively, grew steadily over time. Nevertheless, 115 some clones behaved unpredictably, with proportions varying by mutant gene. Most notable 116 were JAK2-V617F-mutant clones, for which growth trajectories were particularly erratic, with 117 only $58 \%$ displaying stable growth. The likelihood of mutant clones displaying non-constant 118 growth at older age was not related to the number of mutations in the same individual 119 ( $p=0.68$; Extended Data Fig. 4d).

120 We further assessed the consistency of clonal trajectories by testing our ability to predict 121 future clonal growth. Using additional prospectively-obtained blood samples from 11 122 individuals, we compared observed versus predicted VAFs (Extended Data Fig. 4e-g, 123 Supplementary Table 4) and found good concordance (mean absolute error: 3.5\%), 124 corroborating our model and providing further evidence that fixed-rate growth is the norm in 125 old age. 
a
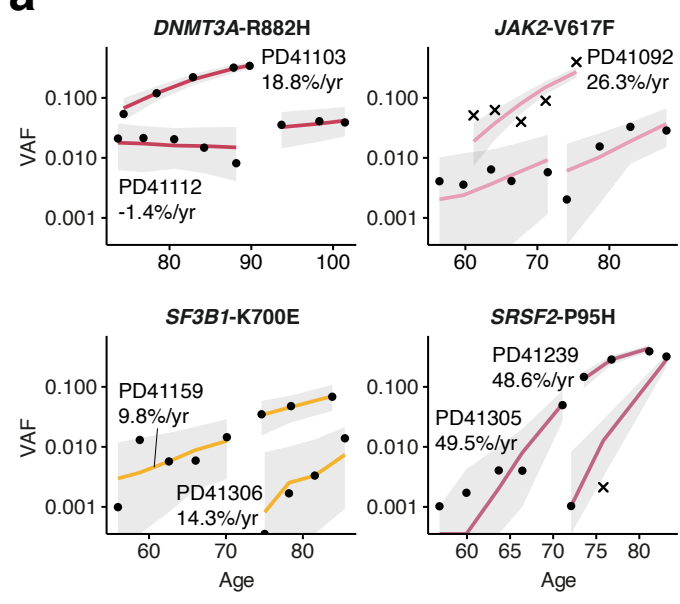

- Logistic fit with constant growth rate $(95 \% \mathrm{Cl})$

\section{C}

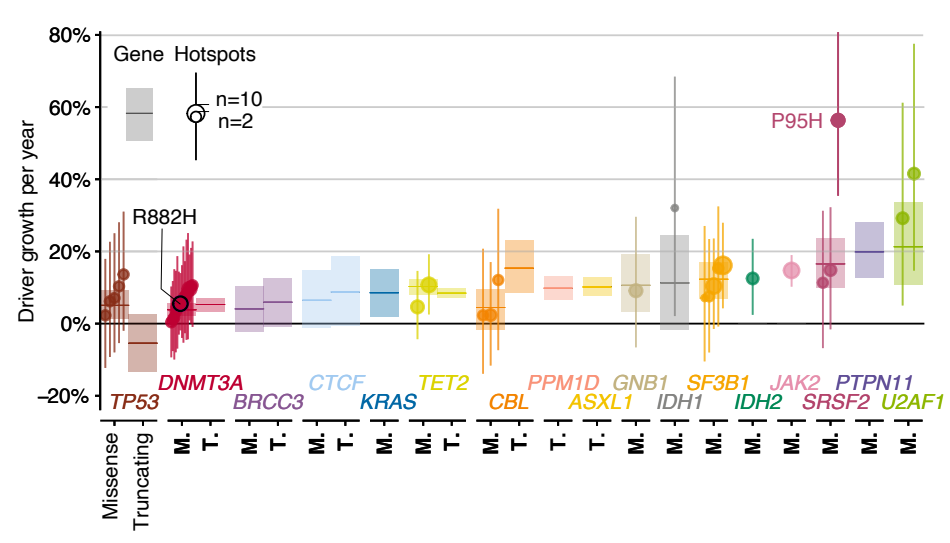

b

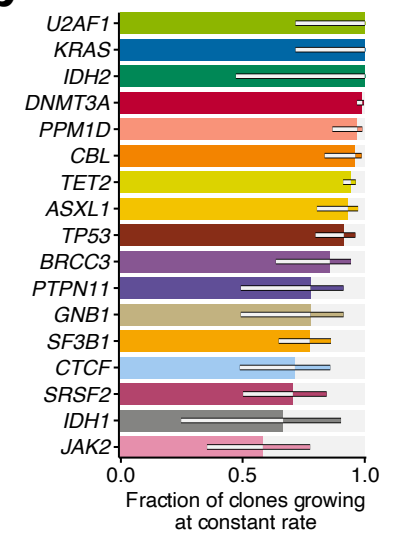

d
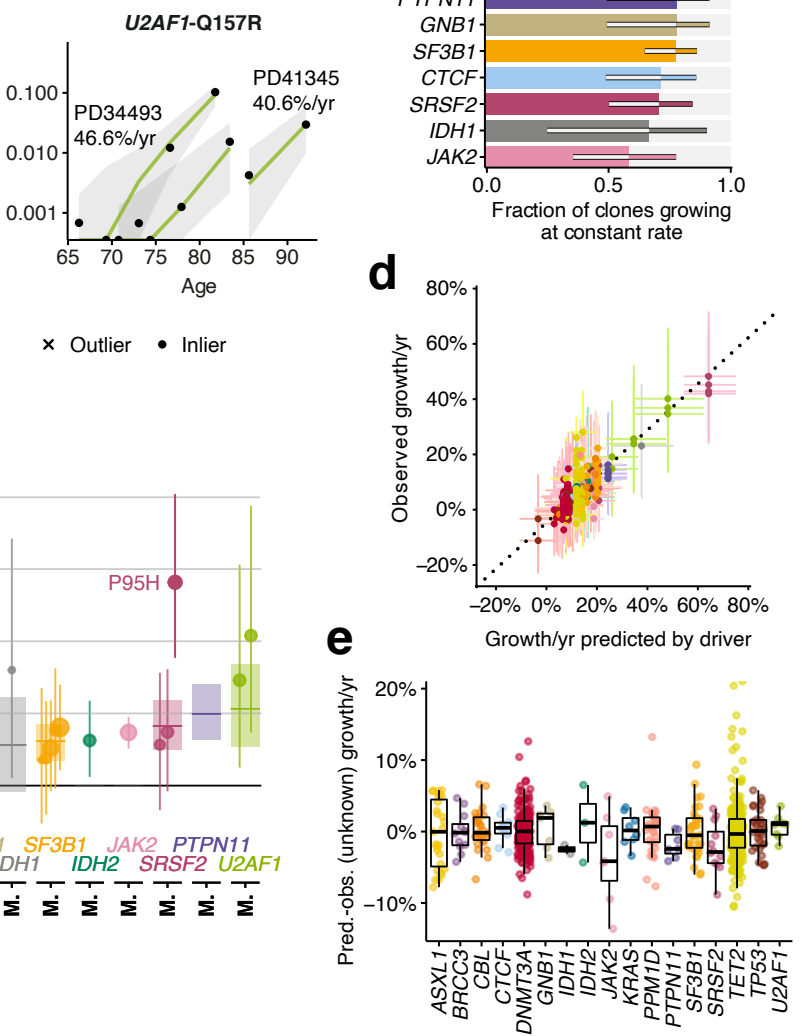

Fig. 2: The longitudinal dynamics of $\mathbf{C H}$ in older age. a, Examples of fitted exponential growth of clones with mutations at 6 common hotspots. Grey bands represent the $95 \%$ highest posterior density interval (HPDI). Each data point is represented by a dot if it conforms to our model of fixed-rate exponential growth and by a cross otherwise (outlier, defined as tail probability less than $2.5 \%$ ). b, Proportion of clonal trajectories showing fixedrate exponential growth, ie. those with no outlying data-points as defined in (a), with $90 \%$ confidence intervals. c, Annual clonal growth associated with different driver mutations, for both whole genes and specific mutation sites. For gene-wise growth, truncating and missense mutations are modelled separately for genes where both are enriched. Sites are modelled separately to gene if mutated recurrently within our cohort. Point estimates for growth and $90 \% \mathrm{HDPI}$ are represented for each site (dot and line, respectively, with dot size proportional recurrence) and each gene (horizontal line and rectangle, respectively). d, Relationship between clonal growth predicted by the identity of the driver mutation and actual observed growth, with $90 \%$ HDPI represented by vertical and horizontal lines, respectively. Vertical spread thereby captures differences in growth rate between clones bearing the same driver mutation. Clones growing faster than predicted lie above the dashed line, and slower clones lie below. e, Distribution of the unknown-cause effect for different genes. Each point represents a single clone and boxplots represent the distribution of these effects for each gene. The value of unknown-cause growth is positive for clones growing faster than expected by the identity of the driver mutation, and negative for clones growing slower than expected.

\section{Determinants of clonal growth rate}

145 To delineate the factors that determine each clone's growth rate, our logistic regression model fits the following contributions of the driver mutation: i) mutated gene; ii) specific amino acid change (for recurrently mutated sites) and iii) mutation type (truncating versus nontruncating) (Supplementary Table 5). An additional component in our model, measuring 
149 variation not captured by (i-iii), was also used and termed "unknown-cause growth" 150 (Extended Data Fig. 4h).

151 We found that clones bearing mutations in different genes expanded at different rates, with mutations affecting DNMT3A and TP53 displaying the slowest average annual growth rates of $\sim 5 \%$ (Fig. 2c, Supplementary Table 6). Clones with mutations in the other most common driver genes (TET2, ASXL1, PPM1D and SF3B1), expanded at roughly twice this rate, i.e. $\sim 10 \% / y r$. The most rapidly expanding clones were those carrying mutations in SRSF2, PTPN11 and U2AF1, growing at over $15-20 \% / y r$ on average. The only specific mutation displaying distinctive behaviour was SRSF2-P95H, which was associated with significantly faster expansion compared to other SRSF2 mutations. By contrast, all other hotspot mutations drove growth at rates similar to mutations elsewhere in the same gene, including commonly mutated sites such as DNMT3A-R882, SF3B1-K666N and SF3B1-K700E.

For most genes, truncating and missense mutations drove comparable rates of growth. Exceptions were TP53, where missense grew 10\%/yr (90\% $\mathrm{Cl}=[3-18 \%])$ faster than truncating mutations (which usually did not expand or even contracted) and CBL, where missense grew 11\%/yr (90\% Cl=[3-19\%]) slower than truncating mutations (Fig. 2c, Extended Data Fig. 4i, Supplementary Table 6).

To quantify the impact of factors other than driver mutations, we compared the observed growth rate of each clone with that predicted by the mutation (Fig. 2d). In Figure 2d, vertical spread thereby represents the variability in growth rate between distinct clones with the same driver mutation. On average, this unknown-cause growth contributed approximately $+/-$ $5 \% / y r$ to clonal expansion (Fig. 2e). Consequently, for fast-growing clones, including those associated with SRSF2-P95H or mutant U2AF1, this effect was proportionately small and there was relatively little inter-individual variability in growth rate. By contrast, the impact on "slow" drivers, such as DNMT3A, was more substantial, with some clones growing twice as fast as predicted by the mutation, and others showing negligible expansion. Clones harbouring JAK2-V617F mutations were an exception as they displayed an unusually high degree of inter-individual variability in relation to average growth rate (Fig. 2d,e, Extended Data Fig. 5a). In view of the well-described heritable contribution to myeloproliferative neoplasm (MPN) susceptibility ${ }^{22,23}$, we tested if JAK2-V617F-mutant clones grew faster in individuals with inherited MPN risk alleles, but found no such relationship (Extended Data Fig. 5b, Supplementary Table 7). However, we also made the more general observation that certain individuals harboured more mutations in the same gene than would be expected by chance (Extended Data Fig. 5c), suggesting that non-mutation factors influencing clonal growth are both individual- and gene-specific. While we found no evidence that these nonmutation factors include either sex or smoking history, since neither accounted for differences in clonal growth rate between individuals with the same mutant driver gene, age was a significant factor specifically for TET2-mutant clones, which grew faster in older individuals (Spearman's rho $=0.31$; adj. p-value $=2.33^{\star} 10^{-6}$ ) (Extended Data Fig. $5 d-f$ ).

\section{Haematopoietic phylogenies give insights into the lifelong natural history of $\mathrm{CH}$}

190 To contrast the longitudinal clonal behaviours we observed in older age with lifelong clonal 191 dynamics, we began by deriving and whole-genome sequencing (WGS) 96 single-cell- 
192 derived colonies from each of three individuals with splicing gene mutations (Fig. 3a-c), 193 particularly as previous reports suggested a possible interaction of these mutations with age ${ }^{8}$. 194 We constructed phylogenetic trees using somatic mutations as lineage-tracing barcodes 195 and, since HSCs accumulate mutations at a near constant rate, we used phylogenetic branch 196 lengths to time the onset of clonal expansions ("clades") ${ }^{28-31}$. In PD41276, the phylogeny was 197 dominated by an SF3B1-K666N-mutant clone, beginning between 23-47 years of age, with 198 only a single SF3B1-wild type colony, consistent with a near-complete clonal sweep (Fig. 3a). 199 In PD34493, SF3B1-K666N was acquired prior to the age of 35 years, whilst U2AF1-Q157R 200 initiated clonal growth later (age 41-61) in a previously expanded clade lacking recognisable 201 drivers (Fig. 3b). Interestingly, an additional apparently driverless expansion - a phenomenon 202 recognised in old age $\mathrm{e}^{6,32}$ - was observed in this individual (Fig. 3b), and a further 3 such 203 expansions in PD41305 (Fig. 3c). In PD41305, the SRSF2-P95H mutation was present in only 204 one colony, preventing characterisation of its phylogeny beyond the observation that it was 205 acquired after the age of 13 years (Fig. 3c).

206 We next used the timing and density of clonal branchings (or "coalescences") to reconstruct 207 the entire growth trajectories of expanded clades using phylodynamic principles (Fig. 3d$208 \mathrm{~h}$ ) $29,33,34$. This revealed that the three clades with identified drivers (SF3B1-K666N and U2AF1209 Q157R in PD34493, and SF3B1-K666N in PD41276), expanded (Fig. 3d-f) at calculated rates 210 similar to those observed in our time-series VAF measurements during older age (Fig. 3i, left 211 panel). Of note, SF3B1-K666N was associated with a substantially different growth rate in 212 PD41276, where it expanded at 28\%/yr by serial VAFs (29\%/yr by phylodynamic estimate), 213 versus 10\%/yr in PD34493 (17\%/yr by phylodynamics) (Fig. 3i). Reasons for this difference 214 are unclear, but it is notable that the faster-growing clone had antecedent $Y$ loss (Fig. 3a), 215 an aberration seen in clades from all three individuals and associated with only modest clonal 216 expansion when isolated (Fig. 3a-c). Interestingly, clones without known drivers began to 217 expand within the first two decades of life and grew over their lifetimes at rates comparable 218 to clones with known drivers (14-32\%/yr) (Fig. 3g,h, Extended data Fig. 6). 
a

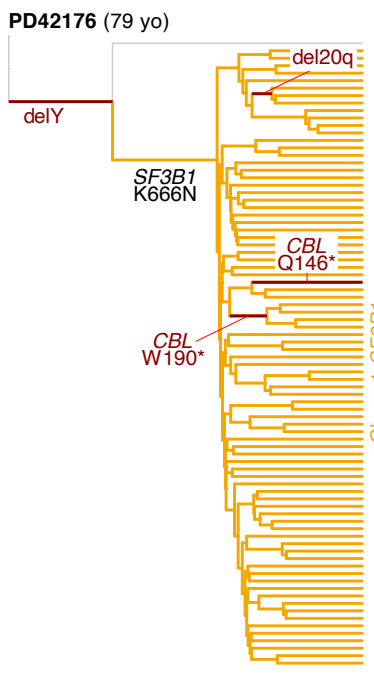

d
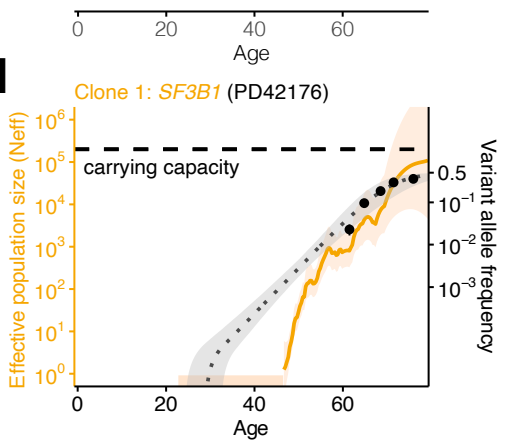

i

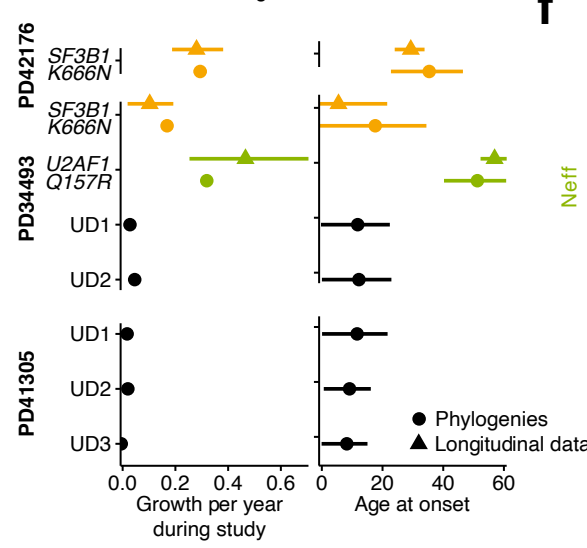

b
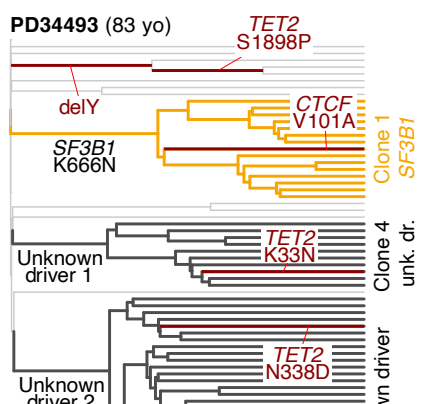

Unknown
driver 2

e

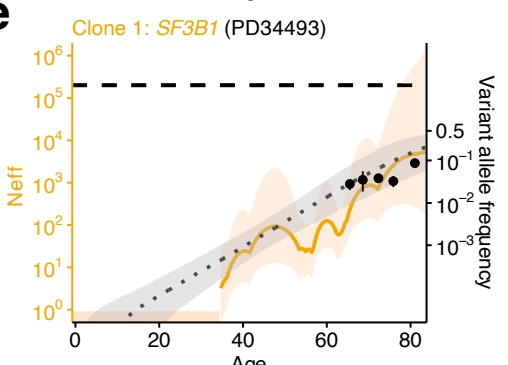

f

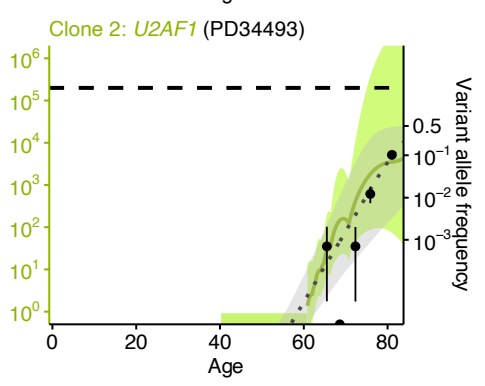

Inferred clone size (mean and $95 \% \mathrm{Cl}$ )
C PD41305 (73 уо)

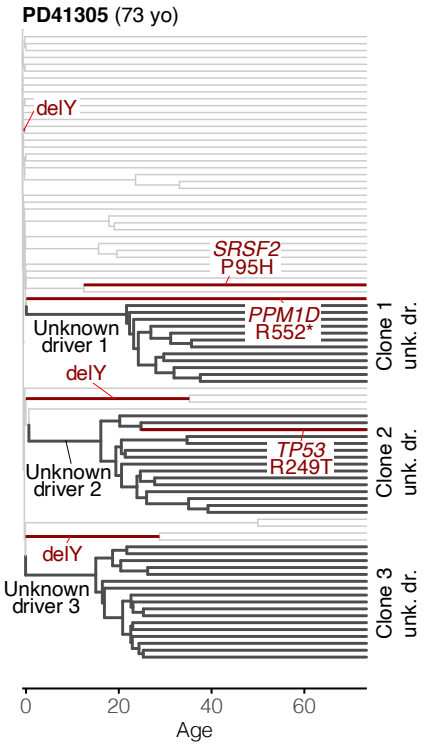

g

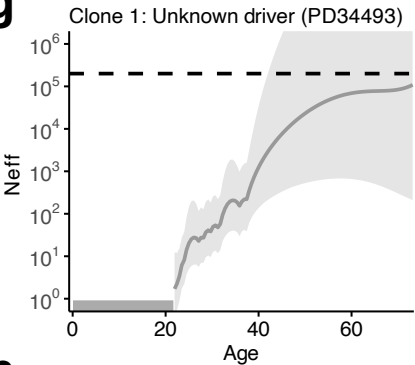

h
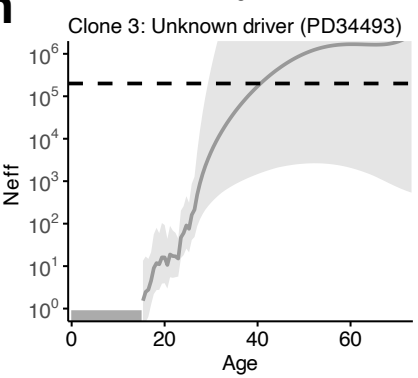

Variant allele frequency

from serial blood samples and $90 \% \mathrm{Cl}$

Evolutionary model fit

Fig. 3: Haematopoietic phylogenetic trees. a-c, Haematopoietic phylogenies of participants PD41276 (a), PD34493 (b) and PD41305 (c). Each tree tip is a single cell-derived colony and tips with shared mutations coalesce to an ancestral branch, from which all colonies in such a "clade" arose. Branch lengths are proportional to the number of somatic mutations, which accumulate linearly with age. Branches containing known driver mutations or chromosomal aberrations are annotated. Clonal expansions are coloured: SF3B1-K666N-mutant expansions in orange, U2AF1-Q157R-mutant expansions in green, and expansions without identified drivers ('Unknown driver' or 'UD') in black. d-h, Growth trajectories of each clonal expansion, as determined by (i) phylogenies (effective population size (Neff) estimated using phylodynamic methods), and (ii) time-series data (using serial VAF measurements and modelled historical growth, as illustrated in Fig. 2, if available). Phylogeny-derived age at clone onset range is represented as a horizontal coloured bar on the x-axis, with the limits of the bar corresponding to the age range of the phylogeny branch along which the corresponding driver mutation was acquired. $\mathbf{i}$, Comparison of the ages at onset (right) and growth rate during study period (left) derived from phylogenetic trees and longitudinal data.

\section{Many clones decelerate before older age}

234 As the phylodynamic reconstruction of a clone goes back to its inception, we investigated whether clonal growth dynamics during earlier life deviate from the stable growth observed 
during older age. To corroborate observations from the three individuals depicted in Fig. 3, we conducted additional phylodynamic analyses of trees derived from 1,461 whole-genome sequenced single cell-derived colonies from another four individuals aged 75-81yrs from the study by Mitchell et al. ${ }^{32}$. This revealed that, in many instances, the reconstructed effective population size (Neff) of any individual clone grew more slowly towards the sampling date, before it saturated the HSC compartment (Fig. 4a-b; Extended Data Fig. 7a-c). This characteristic deceleration was quantified by fitting a biphasic exponential growth model to early and late parts of the trajectories (Fig. 4c). In most cases, extrapolating early growth (a consistent estimator of the fitness advantage of a clone in Wright-Fisher simulations, Extended Data Fig. 7d, Extended Data Fig. 8) led to dramatic overestimations of clade size (median 35x; Fig. 4d, Extended Data Fig. 7e).

247 We used our longitudinal cohort to orthogonally test the lifelong stability of clonal growth by extrapolating the observed (fitted) trajectory of each clone backwards in time to infer the age at clonal onset. To account for stochastic drift, which can lead to faster growth of small clones, and the finite carrying capacity of the HSC population, which naturally limits/slows large clones, we derived and used an approximation to a Wright-Fisher process (Extended Data Fig. 4a,b). While estimates of age at clonal onset agreed with phylogenetic estimates for the fast-growing splice factor mutations (Fig. 3i), for many other clones, constant lifelong growth at the rate we observed during old age would be too slow to explain the observed VAFs (Fig. 4e,f,g), proposing that clonal expansion was faster in earlier life. These observations reveal that, at least for some clones/genes, the dynamics observed in later life are not representative of those that prevail earlier.

258 We then assessed the minimum lifetime rate at which clones must have grown in order to reach the observed VAFs in our longitudinal data, henceforth termed 'historical growth', by restricting fits/solutions to growth rates that would place the age of clonal onset within individuals' lifetimes (Fig. 4h, Supplementary Table 8). Expectedly, this minimal historical growth rate was typically higher than the growth rate observed during the study period (i.e. in older age; Fig. 4i, Extended Data Fig. 7f). Moreover, the fold-changes between historical and observed growth rates derived from longitudinal data were qualitatively in good agreement with the fold-changes between late growth and expected growth (the latter assuming growth is constant through life and carrying capacity is fixed) derived from phylodynamic data (Fig. 4c,i, Extended Data Fig. 7f). Taken together it thus emerges that many clones grew more rapidly early in life compared with the rate in old age. 
a

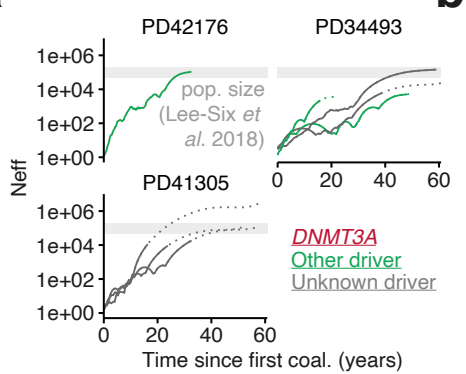

b

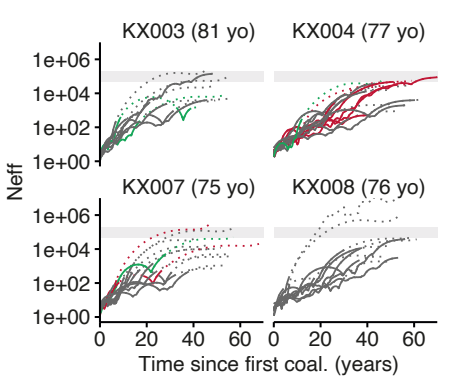

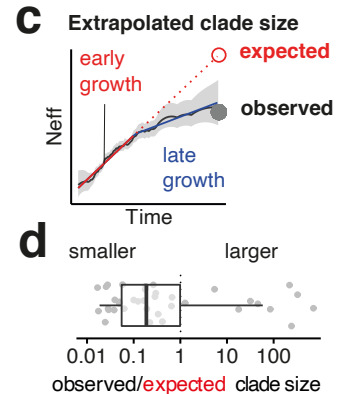

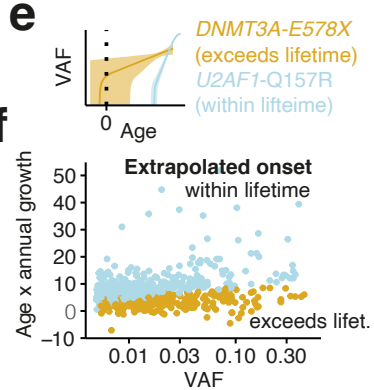

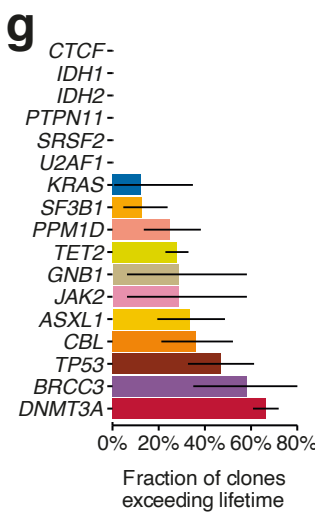
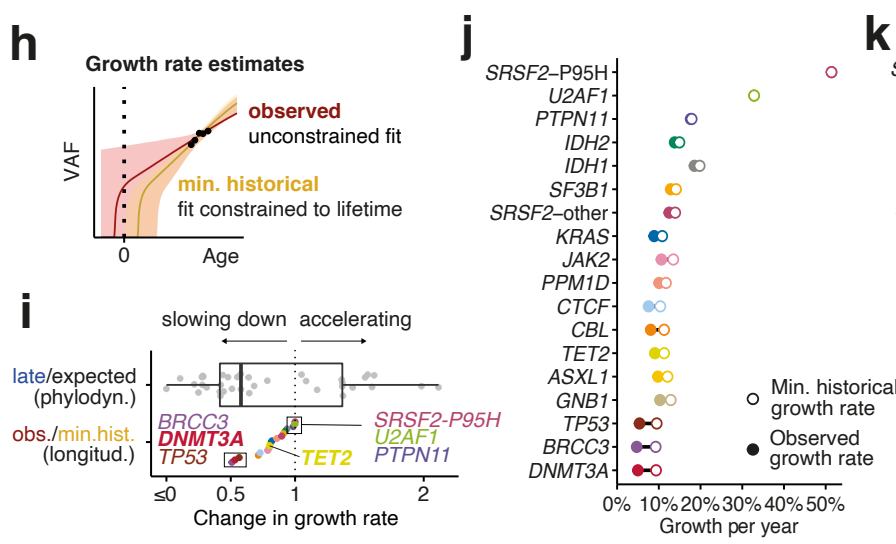

k

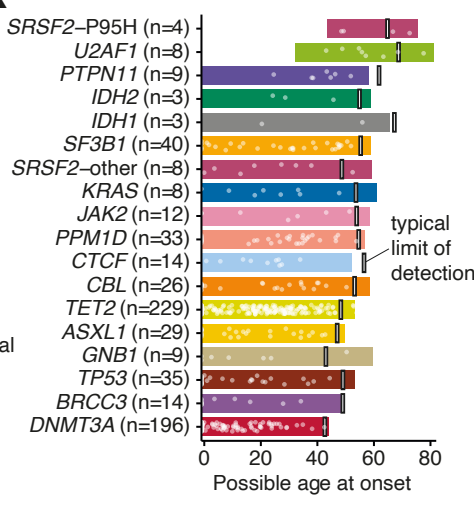

Fig. 4: Evidence for clonal deceleration from single-cell phylogenies and longitudinal data. a,b. Effective population size (Neff) trajectories inferred from single cell phylogenies in this paper (a) and in Mitchell et $\mathrm{a}^{32}(\mathrm{~b})$. Dotted lines represent parts of the trajectory with high variance $(\log (\operatorname{var}(\operatorname{Neff}))>5)$. c. Representation of biphasic fit to Neff estimates and extrapolation from early growth (observed clone size is calculated as the clonal fraction in the phylogeny scaled by an Neff of 200,000 HSC x yr; comparison with 1,000,000 HSC x yr in Extended Data Fig. 7e). d. Ratio between observed and expected (extrapolated from early growth) clone size from phylogenies. e. Representation of extrapolated trajectories derived from longitudinal data, assuming stable lifelong growth at the same fixed rate we observed during older age; some projections are not feasible (ie. exceeding lifetime, with onset pre-conception). f. Relationship between age and the observed growth rate of clones and VAF (longitudinal data; light blue represents clones with projected onset within lifetime and golden represents those exceeding lifetime). g. Quantification of unfeasible clones (exceeding lifetime) per gene (longitudinal data). $\mathbf{h}$. Representation of the calculation of minimum historical growth. i. Quantification of the ratios between observed and historical (longitudinal data) and between late and expected (phylogenetic data) growth. j. Differences between the median observed and historical growth per year for each gene. k. Projected ages at onset for all clones, assuming stable lifelong growth at the same fixed rate we observed during older age.

\section{Driver-specific differences in lifetime clonal behaviour}

The effect of deceleration was most marked for clones bearing mutations in DNMT3A, $B R C C 3$ and TP53, whose early growth was at least twice as fast as that measured during old age (Fig. 4i,j). Conversely, we observed almost no deceleration of fast-growing clones harbouring U2AF1, SRSF2-P95H, PTPN11 or IDH1 mutations (Fig. 4i,j). It is particularly notable that the TET2-mutant clones were much less susceptible to deceleration than DNMT3A-mutant clones (Fig. 4i-j). This is consistent with the observation that the prevalence of TET2-mutant $\mathrm{CH}$ rises at older ages and eventually exceeds that of DNMT3A-mutant $\mathrm{CH}$, which is more prevalent at younger ages (Fig. 1d). A declining relative advantage of DNMT3A mutations in older age was also suggested by the much lower proportion of DNMT3A mutantclones reaching detectable limits during our study period compared to clones bearing mutations in other genes ("incipient clones", Extended Data Fig. 9a). 
To derive representative ranges for age at clone onset for each driver gene, we capped individual estimates at conception, thus avoiding estimates that projected beyond individuals' lifetimes (Fig. 4k, Extended Data Fig. 9b,c). We also validate this method using

300 simulations and confirm that these ranges are not affected by changes in Neff or generation

301 time (Extended Data Fig. 9d,e). We estimated that the average latency between clone

302 foundation and detection in peripheral blood at VAF $\geq 0.2 \%$ (Supplementary Note 1) was 30

303 years across all clones, with considerable variability between mutant genes, ranging from 38

years for DNMT3A-mutant clones to 12 years for U2AF1-mutant clones. Most drivers were projected to initiate expansions of clones throughout life, compatible with the notion that somatic mutations occur at a constant rate ${ }^{28,29,35}$. However, solutions for DNMT3A-mutant clones concentrated earlier in life, consistent with early initiation and rapid expansion followed by marked deceleration then slow growth, as discussed earlier. Of note, capping onset at conception is arbitrary and it remains possible that some clones start later and exhibit faster initial growth followed by even stronger deceleration, a scenario that would be more consistent with published fitness estimates of $11-19 \% / \mathrm{yr}$ based on cross-sectional VAF measurements $^{12}$. In contrast, SRSF2-P95H and U2AF1 mutations initiated clonal expansion always after 30 years of age and with a median age at onset of 58 and 57 years, respectively

314 (Fig. 4k). This indicates that the reported rarity of these mutant clones in people aged $<60$ years ${ }^{5,6,8}$ is not due to slow growth over decades, but rather due to their late onset followed by rapid expansion and also provides a plausible explanation for the high risk of leukaemic

\section{8} progression associated with these mutations ${ }^{9,36}$.

319 To investigate the links between mutation fitness and malignant progression, we built on our 320 previous study of $\mathrm{AML}$ risk prediction ${ }^{9}$ and revealed that among $\mathrm{CH}$ driver genes a faster growth rate was associated with a higher $A M L$ risk (adjusted $R^{2}=0.55, p=0.0037$, Fig. 5a). For example, genes driving fast $\mathrm{CH}$ growth like SRSF2 and U2AF1 were associated with the highest risks of leukaemogenesis, while slow-growing clones such as those bearing DNMT3A mutations, conferred a lower risk. To confirm our findings in larger studies and include myeloid malignancies other than AML, we analysed large published datasets of $\mathrm{AML}$ $(n=1540)^{37}$ and myelodysplastic syndromes (MDS, $\left.n=738\right)^{38}$ using a site-specific extension of the dNdScv algorithm to formally quantify the extent to which individual hotspots are under the influence of positive selection in these cancers (Supplementary Tables 9,10) ${ }^{25}$. This analysis revealed a positive correlation between each hotspot's growth coefficient in $\mathrm{CH}$ and its selection strength in myeloid cancer (Fig. $5 b$; adjusted $R^{2}=0.19, p=0.0016$ ), corroborating the AML risk analysis. Nevertheless, the observation that the same $\mathrm{CH}$ driver gene can progress to either AML or MDS, with variable predilections as quantified by gene-level dN/dS comparison (Extended Data Fig. 10; Supplementary Table 10), suggests that factors other than growth rate can also influence a mutation's malignant potential. 


\section{a}

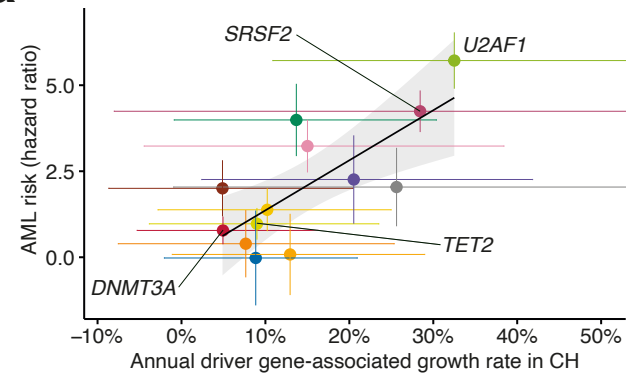

b

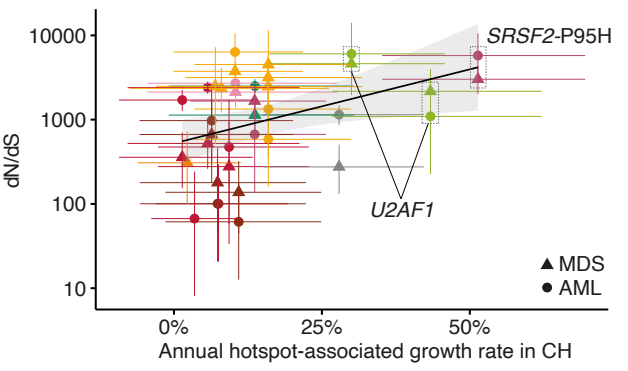

Fig. 5: CH dynamics and progression to myeloid disease. a, Relationship between the growth rate associated with each driver gene in $\mathrm{CH}$, and the risk of $\mathrm{AML}$ progression associated with that driver gene. $\mathbf{b}$, Relationship between the growth rate associated with each recurrent mutation in $\mathrm{CH}$, and the strength of selection of that mutation in AML (circles) and MDS (triangles). In $\mathbf{a}$ and $\mathbf{b}$ genes/hotspots mentioned in the main text are highlighted.

\section{Discussion}

343 The phenomenon of $\mathrm{CH}$ has served as an exemplar in the developing understanding of somatic mutation, clonal selection and oncogenesis in human tissues ${ }^{4,39}$. However, the nature of these interrelated processes can change over time and their consequences develop only slowly, making them difficult to investigate. Here, we studied the longitudinal behaviour of $\mathrm{CH}$ over long periods (median 13 years) and combined this with lifelong phylodynamic analyses of haematopoiesis to derive new insights into these fundamental biological processes.

350 First, we found that most clones (92\%) display stable exponential growth dynamics in older age, at rates influenced by their driver mutations. This allowed us to predict future clonal growth trajectories, a finding with potentially useful implications for clinical practice (Extended Data Fig. 4e-g). Surprisingly, mutations in DNMT3A, reportedly the most common $\mathrm{CH}$ driver gene $\mathrm{e}^{5-7}$, were associated with slower clonal expansion than most other $\mathrm{CH}$ genes. Also, DNMT3A hotspot mutations (e.g. at codon R882) were not associated with faster growth than other DNMT3A mutations (Figure 2c). By contrast, TET2-mutant clones expanded significantly faster over the study period (Fig. 2c) and, reflecting this, also reached detectable levels much more frequently on-study than DNMT3A-mutant clones (Extended Data Fig. 9a). This resulted in TET2 becoming the most prevalent $\mathrm{CH}$ driver after the age of 75 years (Figure $1 d$ ).

361 These initial findings suggested that, while clonal growth is remarkably stable in old age, dynamics in earlier life may deviate from this behaviour, challenging the premise that mutation fitness is constant over the human lifespan ${ }^{12}$. To test this, we first attempted to derive when individual $\mathrm{CH}$ clones were founded, using simple retrograde extrapolation of observed trajectories. This led to projected ages at clonal foundation that preceded conception for a 


\section{Methods}

\section{Study participants}

404 Ethical permission for this study was granted by The East of England (Essex) Research Ethics

large number of clones (Fig. 4f,g), implying that their early growth must have been faster than that we observed during old age. This was most striking for DNMT3A, for which more than two thirds of projections were implausible (ie. onset pre-conception), but less common for TET2 and very uncommon for splicing factor genes (Fig. 4g).

To further investigate lifelong clonal behaviour, we analysed haematopoietic phylogenies from healthy old individuals and found that aged haematopoiesis was dominated by a small number of expanded HSC clones, some of which lacked recognisable drivers ${ }^{32}$. Using phylodynamic approaches to track clonal growth rates through life, in conjunction with findings from our longitudinal cohort, we reveal widespread clonal deceleration prior to the period of stable growth during old age, in the context of an increasingly competitive oligoclonal HSC compartment (Fig. 4i). DNMT3A-mutant clones, as well as those bearing mutations in TP53 and BRCC3 and also apparently driverless clones, were among those displaying the most marked degree of deceleration (Fig. 4i). In contrast, TET2 mutations appeared to drive more stable lifelong growth (Fig. 4h-j), which may underlie their apparent ability to initiate clonal expansion fairly uniformly through life (Fig. $4 \mathrm{k}$ ) and the fact that TET2 "overtakes" DNMT3A as the most common $\mathrm{CH}$ driver after the age of 75 years (Fig. 1d).

In diametric contrast to $D N M T 3 A$ and unlike other genes, $\mathrm{CH}$ driven by mutant $U 2 A F 1$ and SRSF2-P95H only initiated late in life (Fig. 4k) and exhibited some of the fastest expansion dynamics (Fig. 2c). These data were corroborated by phylogenetic analyses (Fig. 3b,f) and tally with the sharp increase in prevalence of splice factor-mutant $\mathrm{CH}^{8}, \mathrm{MDS}^{38,40,41}$ and $\mathrm{AML}^{37,42}$ in old age and the high risk of progression to myeloid cancers associated with these mutations ${ }^{9}$. The particular behaviour of these clones proposes a specific interaction with ageing, which could relate to cell-intrinsic factors or to cell-extrinsic changes in the aging haematopoietic niche that make it more suitable for HSCs harbouring splice factor mutations ${ }^{43,44}$.

Finally, we explored the relationship between clonal growth rate in $\mathrm{CH}$ and the development of myeloid cancers. We find that mutations associated with faster $\mathrm{CH}$ growth are also those associated with higher risk of progression to AML (Fig. 5a) and are under the strongest selective pressure in AML and MDS (Fig. 5b). Indeed, we show that the average annual growth per gene explains over $50 \%$ of the variance in $\mathrm{AML}$ risk progression. This shows that an improved understanding of growth dynamics in $\mathrm{CH}$ can help identify those at risk of myeloid malignancies.

Collectively, our work gives new insights into the lifelong clonal dynamics of different subtypes of $\mathrm{CH}$, the impact of ageing on haematopoiesis, and the processes linking somatic mutation, clonal expansion and malignant progression. Committee (REC reference 15/EE/0327). The SardiNIA longitudinal study recruited individuals from four towns in the Lanusei Valley in Sardinia, capturing 5 phases of sample and data 
collection over more than 20 years ${ }^{25}$. We analysed serial samples from 385 individuals in the

408 SardiNIA project.

\section{Targeted sequencing and variant-calling}

410 Target enrichment of genomic DNA was performed using a custom RNA bait set (Agilent 411 SureSelect ELID 3156971), designed complementary to 56 genes implicated in $\mathrm{CH}$ and 412 haematological malignancies (Supplementary Table 1). Libraries were sequenced on Illumina 413 HiSeq 2000 and variant-calling was performed as we described previously ${ }^{9,45}$. Briefly, somatic 414 single-nucleotide variants and small indels were called using Shearwater (v.1.21.5), an 415 algorithm designed to detect subclonal mutations in deep sequencing experiments ${ }^{46}$. Two 416 additional variant-calling algorithms were applied to complement this approach: CaVEMan 417 (v.1.11.2) for single-nucleotide variants, and Pindel (v.2.2) for small indels ${ }^{47,48}$. VAF correction 418 was performed using an in-house script (https://github.com/cancerit/vafCorrect). Finally, 419 allele counts at recurrent mutation hotspots were verified using an in-house script 420 (github.com/cancerit/allelecount). Variants were filtered as we described previously,45, but 421 were not curated with regard to existing notions of oncogenicity, ie. all somatic variants 422 passing quality filters were retained for analysis.

If a variant was identified in an individual at any time-point in the study, this site was requeried in the same individual at all other time-points, using an in-house script (cgpVAF) to provide pileup (SNV) and Exonerate (indel) output (https://github.com/cancerit/vafCorrect). No additional filters were applied to these back-called variants.

\section{Selection analyses (dN/dS)}

429

To quantify selection, we used the dNdScv algorithm, a maximum-likelihood implementation of $\mathrm{dN} / \mathrm{dS}$, which measures the ratio of non-synonymous $(\mathrm{N})$ to synonymous $(\mathrm{S})$ mutations, while controlling for gene sequence composition and variable substitution rates ${ }^{26}$. We first applied this method to the mutation calls from the longitudinal SardiNIA cohort in order to identify which genes are under positive selection in the context of $\mathrm{CH}$. For this analysis, any mutation that was present in a single individual at multiple time-points was counted only once.

To characterise patterns of selection in AML and MDS, we applied dNdScv to two published data sets. The AML set was derived from 1540 patients enrolled in three prospective trials of intensive therapy ${ }^{37}$. The MDS set included 738 patients with MDS or closely related neoplasms such as chronic myelomonocytic leukaemia ${ }^{38}$. Both used deep targeted sequencing of 111 cancer genes, which overlapped with 13 of the 17 genes of interest in our longitudinal $\mathrm{CH}$ study (PPM1D, CTCF, GNB1 and BRCC3 were not sequenced in the AML/MDS studies). We called and filtered variants in the 13 overlapping genes using the strategy described above (under 'Targeted sequencing and variant-calling'). Variants were identified in all 13 genes in both AML and MDS datasets (Supplementary Table 10). We calculated dN/dS values both at the level of individual genes, and at single-site level for hotspots, the latter using the sitednds function in the dNdScv R package.

\section{Hierarchical modelling of clone trajectories through time}

447 We use Bayesian hierarchical modelling to model clonal trajectories. Since we are unable to 448 phase different mutations into specific clones and given that individual $\mathrm{CH}$ clones typically 
449

450

451

452

453

454

455

456

457

458

459

460

461

462

463

464

465

466

467

468

469

470

471

472

473

474

475

476

477

478

479

480

481

482

483

484

485

486

487 harbour a single mutation ${ }^{49}$, we assume that each mutation is heterozygous and its VAF is representative of the prevalence of a single clone. Accordingly, for a given individual $j$ and mutation $i$, we have a mutant clone $c_{i j}$. We model the counts $\operatorname{counts}_{c_{i j}}$ for $c_{i j}$ at age $t$ as a binomial distribution, such that $\operatorname{counts}_{c_{i j}}(t) \sim \operatorname{Bin}\left(\operatorname{cov}_{i j}(t), p_{i j}(t)\right)$, with $\operatorname{cov}_{i j}$ as the coverage of this mutation at age $t$ and $p_{i j}(t) \sim B \operatorname{Beta}(\alpha(t), \beta)$ as the expected proportion of mutant allele copies. As such, $\operatorname{counts}_{c_{i j}}(t) \sim B B\left(\operatorname{cov}_{i j}(t), \alpha(t), \beta\right)$. Here, $\beta \sim N\left(\mu_{o d}, \sigma_{o d}\right)$ is the technical overdispersion whose parameters are estimated using replicate data (details below) and $\alpha(t)=\frac{\beta q(t)}{1-q(t)}$, where $q(t)=\operatorname{ilogit}\left(\left(b_{\text {gene }_{i}}+b_{\text {site }_{i}}+b_{c_{i j}}\right) * t+u_{i j}\right)$. We use this parameterization to guarantee that $E\left[\operatorname{counts}_{c_{i j}}\right]=p_{i j} \operatorname{cov}_{i j} . b_{\text {gene }_{i}} \sim N(0,0.1)$ and $b_{\text {site }_{i}} \sim$ $N(0,0.1)$ are the gene and site growth effects for mutation $i$, respectively. $b_{c_{i j}} \sim N(0,0.05)$ is the growth effect associated exclusively with mutation $i$ in individual $j$ - i.e. of mutant clone $c_{i j}$ - and $u_{i j}$ is the offset accounting for the onset of different clones at different points in time. We also define the growth effect of $c_{i j}$ as $b_{\text {total }_{i j}}=\left(b_{\text {gene }_{i}}+b_{\text {site }_{i}}+b_{u c_{i j}}\right)$. Along this work we will refer to $b_{\text {gene }_{i}}+b_{\text {site }_{i}}$ as the driver (growth) effect and to $b_{c_{i j}}$ as the unknown-cause (growth) effect - the fraction of growth that is quantifiable but not explained by either gene or site.

Preventing identifiability issues and reducing uninformed estimates. To address possible identifiability issues in our model, when a gene has a single mutation (JAK2-V617F and IDH2$\mathrm{R} 140 \mathrm{Q}$ ), the effect is considered to occur only at the site level. To avoid estimating the dynamics of a site from a single individual, we only model $b_{\text {site }}$ when two or more individuals have a missense mutation on site $i$ - we refer to these sites as "recurrent sites". Overall, we consider a total of 17 genes and 39 recurrent sites (Supplementary Table 5).

Estimating and validating growth parameters. Using the model described above, we use Markov Chain Monte Carlo (MCMC) with a Hamiltonian Monte Carlo (HMC) sampler with 150300 leapfrog steps as implemented in greta ${ }^{50}$. We sample for 5,000 iterations and discard the initial 2,500 to get estimates for the distribution of our parameters. As such, our estimates for each parameter are obtained considering their mean, median and $95 \%$ highest density posterior interval for 2,500 samples.

We assess the goodness-of-fit using the number of outliers detected in any trajectory and consider only trajectories with no outliers as being explained by our model and, as such, growing at constant rate. Outliers are assessed by calculating the tail probabilities of the counts under our model with a hard cut-off at $2.5 \%$. As such, $P_{\text {outlier }}=1$ if $P\left(\right.$ counts $\left.\mid b_{\text {gene }_{i}}, b_{\text {site }_{i}}, b_{c_{i j}}, u_{i j}, t\right)<0.025 \mid P\left(\right.$ counts $\left.\mid b_{\text {gene }_{i}}, b_{\text {site }_{i}}, b_{c_{i j}}, u_{i j}, t\right)>0.975 \quad$ and $P_{\text {outlier }}=0$ otherwise. We validate this approach using Wright-Fisher simulations (Supplementary Methods). We additionally assess the predictive power of this model on an additional time-point that was available for a subset of individuals and that was not used in the inference of parameters in our model (Supplementary Methods).

Estimating the technical overdispersion parameter. Technical VAF overdispersion used two distinct sets of data: 
(1) Horizon Tru-Q-1 was serially diluted to VAFs of $0.05,0.02,0.01,0.005$ and 0 using Horizon Tru-Q-0 (verified wild-type at these variant sites), then sequenced in duplicate or triplicate;

(2) 19 SardiNIA samples with mutations across 15 genes at a range of VAFs, were sequenced in triplicate.

Sample processing and analysis was performed as described in the "Targeted Sequencing and Variant-calling" section. Replicate samples were picked from the same stock of DNA, then library preparation and sequencing steps were performed in parallel. Variant calls for these replicate samples are in Supplementary Table 11.

For (1), we model the distribution over the expected $V A F$ as a beta distribution such that $V A F \sim \operatorname{Beta}(\alpha, \beta)$ and for (2) we adopt a model identical to the one described earlier in this section but use only gene growth effects $\left(\operatorname{counts}_{c_{i j}}(t) \sim B B\left(\operatorname{cov}_{i j}(t), \alpha(t), \beta\right), \alpha(t)=\frac{\beta q(t)}{1-q(t)}\right.$, $\left.q(t)=\operatorname{ilogit}\left(b_{\text {gene }_{i}} * t+u_{i j}\right)\right)$. Here, we model $\beta \sim \exp (r)$ with $r$ as a variable with no prior. We use MCMC with HMC sampling with 400-500 leapfrog-steps as implemented in greta ${ }^{50}$ to estimate the mean and standard deviation of $\beta$. For this estimate we use 1,000 samples from the posterior distribution.

\section{Analysis of non-mutation factors as determinants of clonal growth rate}

Inherited polymorphisms and JAK2-mutant clonal growth. The SardiNIA cohort had previously been characterised using two Illumina custom arrays: the Cardio-MetaboChip and the ImmunoChip ${ }^{25}$. Inherited genotypes at 12 loci previously associated with MPN risk were extracted for the 12 individuals with JAK2-V617F mutation ${ }^{22,23}$. The relationship between each individual's total number of inherited risk alleles and JAK2-mutant clonal growth rate was assessed by Pearson's correlation. The 46/1 haplotype, which harbours 4 SNPs in complete linkage disequilibrium, was considered as a single risk allele.

Age, sex and smoking experience. We assess the association between unknown-cause growth and age through the calculation of a Pearson correlation considering all genes, both together and separately while controlling for multiple testing. We also assess the association between unknown-cause growth and sex and smoking history using a multivariate regression where unknown-cause growth is the dependent variable and sex and previous smoking experience are the covariates, while also controlling for age.

\section{Determining the expected age at beginning of clone onset}

We consider that HSC clones grow according to a Wright-Fisher model. According to this, for an initial population of HSC $n / 2$, we can consider two scenarios - that of a single growth process where the time at which the cell first starts growing $t_{0}$ is described as $t_{0}=\frac{\log \left(\frac{1}{n}\right)-u}{b_{\text {total }}}$, or that of a two step growth process, where $t_{0}$ adjusted $=t_{0}+\frac{\log \left(g / b_{\text {total }}\right)}{b_{\text {total }}}-\frac{1}{b_{\text {total }}}$, where $g$ is the number of generations per year. The latter scenario is the one chosen, due to its strong theoretical foundation and previous application to mathematical modelling of cancer evolution ${ }^{51}$. The two regimes that describe it are an initial stochastic growth regime and, once the clone reaches a sufficient population size, a deterministic growth regime. The adjustment made to $t_{0}$ in $t_{0}$ adjusted can be interpreted as first estimating the age at which the clone 
reached the deterministic growth phase $\left(t_{0}+\frac{\log \left(g / b_{\text {total }}\right)}{b_{\text {total }}}\right)$ followed by subtracting the expected time for a clone to overcome its stochastic growth phase $\left(\frac{1}{b_{\text {total }}}\right)$. For both $n$ and $g$ 530 we use the estimates based on ${ }^{29}-n=50,000$ and $g=2$. We validate this approach using 531 simulations (Supplementary Methods) and test the approach against our serial VAF data and verify that changes in $n$ and $g$ do not have a dramatic impact on age at onset estimates by considering a range of values $(n=\{10,000 ; 50,000 ; 100,000 ; 200,000 ; 600,000\}$ and $g=$ $534\{1 ; 2 ; 5 ; 10 ; 13 ; 20\})$.

\section{Derivation of blood colonies and phylogenetic tree construction}

536 Sample preparation and sequencing. We selected 3 individuals with splicing gene mutations 537 from the SardiNIA cohort for detailed blood phylogenetic analysis. Peripheral blood samples 538 were drawn into Lithium-heparin tubes (vacutest, kima, $9 \mathrm{ml}$ ) and buccal samples were taken 539 (Orangene DNA OG-250). Peripheral blood mononuclear cells were isolated from blood and 540 plated at 50,000 cells per $\mathrm{ml}$ in MethoCult 4034 (Stemcell Technologies). After 14 days in 541 culture, 96 single haematopoietic colonies were plucked per individual (total 288 colonies) and lysed in 50 $\mu$ l of RLT lysis buffer (Qiagen).

543 Library preparation for whole genome sequencing (WGS) was performed using our low-input 544 pipeline as previously described ${ }^{52,53}$. $150 \mathrm{bp}$ paired-end sequencing reads were generated 545 using the NovaSeq ${ }^{\circledR} 6000$ platform to a mean sequencing depth of $15 x$ per sample. Reads were aligned to the human reference genome (NCBI build37) using BWA-MEM.

547 Variant-calling and filtering. Single-nucleotide variants (SNVs) and small indels were called 548 against an unmatched reference genome using the in-house pipelines CaVEMan and Pindel, 549 respectively ${ }^{47,48}$. 'Normal contamination of tumour' was set to 0.05 ; otherwise standard 550 settings and filters were applied. For all mutations passing quality filters in at least one sample, in-house software (cgpVAF, https://github.com/cancerit/vafCorrect) was used to produce matrices of variant and normal reads at each mutant site for all colonies from that individual. Copy-number aberrations and structural variants were identified using matchednormal ASCAT ${ }^{54}$ and BRASS (https://github.com/cancerit/BRASS). Low-coverage samples (mean $<4 x$ ) were excluded from downstream analysis ( $n=1$, PD41305). Samples in which the peak density of somatic mutation VAFs was lower than expected for heterozygous changes (in practice $\mathrm{VAF}<0.4$ ) were suspected to be contaminated or mixed colonies, and were also excluded from further analysis ( $n=3$, PD41305; $n=9$, PD41276; $n=3$, PD34493).

Multiple post-hoc filtering steps were then applied to remove germline mutations, recurrent library prep / sequencing artefacts, and in vitro mutations, as described previously ${ }^{55}$ and detailed in custom R scripts (https://github.com/margaretefabre/Clonal_dynamics). Buccal samples were used as an additional filter; mutations were removed if the variant:normal count in the buccal sample was consistent with that expected for a germline mutation ( 0.5 for autosomes and 0.95 for $X Y$ chromosomes, binomial probability $>0.01$ ), and were retained if (i) the variant:normal count in the buccal sample was not consistent with germline (binomial probability $<1 \times 10^{-4}$ ) and (ii) the mutation was not present in either of 2 large SNP databases (1000 Genomes Project and Kaviar) with MAF > 0.001.

568 Phylogenetic tree construction and assignment of mutations back to the tree. These steps 569 were also performed as described previously ${ }^{55}$ and are detailed here: 
570 https://github.com/margaretefabre/Clonal_dynamics. Briefly, samples were assigned a 571 genotype for each mutation site passing filtering steps ('present' $=\geq 2$ variant reads and 572 probability $>0.05$ that counts came from a somatic distribution; 'absent' $=0$ variant reads and 573 depth $\geq 6$; 'unknown' = neither 'absent' nor 'present' criteria met). A genotype matrix of shared 574 mutations was fed into the MPBoot program ${ }^{56}$, which constructs a maximum parsimony 575 phylogenetic tree with bootstrap approximation. The in-house-developed $\mathrm{R}$ package treemut 576 (https://github.com/NickWilliamsSanger/treemut), which uses original count data and a 577 maximum likelihood approach, was then utilised to assign mutations back to individual 578 branches on the tree. Since individual edge length is influenced by the sensitivity of variant579 calling, lengths were scaled by $1 /$ sensitivity, where sensitivity was estimated using the 580 proportion of germline variants called.

581 Reconstruction of population trajectories. Phylogenies were made ultrametric (branch lengths 582 normalised) using a bespoke $\mathrm{R}$ function (make.tree.ultrametric, 583 https://github.com/margaretefabre/Clonal_dynamics/my_functions). Assuming a constant 584 rate of mutation acquisition ${ }^{28,29,35}$, the time axis was scaled linearly, where the root of the tree 585 represents conception, and the tips represent age at sampling. We then analysed population 586 size trajectories by fitting Bayesian nonparametric phylodynamic reconstructions (BNPR) as 587 implemented in the phylodyn R package ${ }^{33,34}$ to clades - sets of samples in a phylogenetic tree 588 sharing a most recent common ancestor (MRCA) - defined by either having a driver mutation 589 on the MRCA or a MRCA branch length that spans more than $10 \%$ of the tree depth and with 5905 tips or more. We also estimated the lower and upper bounds for age at onset of clonal 591 expansion to be the limits of the branch containing the most recent common ancestor.

\section{Deceleration in phylogenies and longitudinal data}

593 We detect deceleration using two different approaches - the ratio between expected and 594 observed clone size using phylodynamic estimates and the ratios between observed and historical (from longitudinal data) and between late and expected (from phylogenetic data), respectively. To obtain the late growth rate we fit a biphasic log-linear model to our phylodynamic estimation of Neff - this enables us to obtain an early and a late growth rate (details in the Supplementary Methods).

599 Expected and observed clone size. The expected clone size is calculated by extrapolating 600 the early growth rate until the age of sampling; having this we can calculate the ratio between 601 expected and observed growth. The ratio between these quantities is then used as a measure 602 of deceleration (details in the Supplementary Methods).

603 Growth ratio in phylogenetic data. The late growth rate is defined as the late growth rate 604 defined in the previous section of the methods. The expected growth rate for the phylogenies 605 is calculated as the growth coefficient for a sigmoidal regression that assumes a population 606 size of 200,000 HSC as the carrying capacity. We then use the ratio between these quantities 607 as a measure of deceleration (1 implies no deceleration; $<1$ implies deceleration).

608 Growth ratio in longitudinal data. The observed growth rate is defined as the growth rate 609 inferred directly from the data. The minimal historical growth is the growth rate estimate 610 obtained by restricting clone initiation to a time after conception (age at at onset $>-1$ ). 
611 Associations between $\mathrm{CH}$ dynamics and (i) AML progression and (ii) selection in 612 MDS and AML

613 To calculate the association between $\mathrm{CH}$ dynamics and AML we used the risk coefficients

614 from our previous work in predicting the onset of $\mathrm{AML}^{9}$, which were calculated by fitting a

615 Cox-proportional hazards model that calculated the risk of AML onset associated with each

616 gene while controlling for age, sex and cohort, and estimate the coefficient of correlation

617 between the expected value of the annual growth for the posterior distribution of each gene

618 (considering gene, site and unknown-cause effects) and the AML progression risk.

619 The association between $\mathrm{CH}$ dynamics and selection in MDS and AML use the dN/dS values

620 calculated with dNdScv as previously described in the methods, using two distinct cohorts 621 from previous studies ${ }^{37,38}$. dN/dS values were calculated for all hotspots and their coefficient 622 of correlation with the expected value of the annual growth for the posterior distribution of 623 each hotspot (also considering gene, site and unknown-cause effects) was calculated.

\section{Statistical analyses}

625 All statistical analyses were conducted using the R software ${ }^{57}-M_{C M C}$ models were fitted 626 using gret ${ }^{50}$ and hypothesis testing, generalised linear models and maximum likelihood fits 627 were performed in base $\mathrm{R}$.

629 This work was funded by a joint grant from the Leukemia and Lymphoma Society (RTF6006630 19) and the Rising Tide Foundation for Clinical Cancer Research (CCR-18-500) and by the 631 Wellcome Trust (WT098051). M.F. is funded by a Wellcome Clinical Research Fellowship 632 (WT098051). J.G.A. is supported by the NIHR Cambridge BRC and their opinions are not 633 necessarily those of the NHS, the NIHR or the Department of Health and Social Care. G.S.V. 634 is funded by a Cancer Research UK Senior Cancer Fellowship (C22324/A23015) and work in 635 his lab is also funded by the European Research Council, Kay Kendall Leukaemia Fund, Blood 636 Cancer UK and the Wellcome Trust. E.F.M. is supported by the Wellcome Trust and Beit 637 Foundation (104064/Z/14/Z) and by the EC H2020. The collection of samples and data from 638 the SardiNIA longitudinal cohort study was supported by the Intramural Research Program 639 of the $\mathrm{NIH}$, National Institute on Aging (NIA) of the National Institute of Health (NIH) with 640 contracts N01-AG-1- 2109 and HHSN271201100005C; and by the European Union's 641 Horizon 2020 Research and Innovation Programme under grant agreement 633964 642 (ImmunoAgeing).

\section{Data availability}

644 The data files necessary to run the analysis in https://github.com/josegcpa/clonal dynamics 645 are freely available at https://doi.org/10.6084/m9.figshare.15029118. All sequencing data 646 have been deposited in the European Genome-phenome Archive (EGA) 647 (https://www.ebi.ac.uk/ega/). Targeted sequencing data have been deposited with EGA 648 accession numbers EGAD00001007682 and EGAD00001007683; WGS data have been 649 deposited with accession number EGAD00001007684. Data from the EGA are accessible for 650 research use only to all bona fide researchers, as assessed by the Data Access Committee 
651 (https://www.ebi.ac.uk/ega/about/access). Data can be accessed by registering for an EGA

652 account and contacting the Data Access Committee.

\section{Code availability}

654 All analyses reported in this study used the statistical software R (v.3.6.3). All R files used for 655 the longitudinal and phylodynamic modelling and validation are publicly available at 656 https://github.com/josegcpa/clonal dynamics. All files used for the construction of 657 phylogenetic trees are publicly available at

658 https://github.com/margaretefabre/Clonal_dynamics.

\section{Author contributions}

660 GV and MG conceived and supervised the study. MF, JGA, MSV carried out analyses and 661 generated data figures. MG and JGA developed and implemented the statistical modelling of 662 clonal dynamics. VO, EF, MM and FC oversaw the SardiNIA cohort. VO, EF, MM, EMcK and 663 FC provided samples and data from the Immunoageing study. AD, JR, CH, JB, MF and GV 664 processed participant samples and performed assays. FA, NW, JN and IM generated 665 computational code used in this paper. EM, MC and PC provided single-cell-derived colony 666 WGS data and helped with data analysis/interpretation.

\section{Competing interests}

668 G.S.V. is a consultant for Astrazeneca and STRM.BIO. The other authors declare no 669 competing interests. 


\section{References}

672 1. Lipschitz, D. A., Udupa, K. B., Milton, K. Y. \& Thompson, C. O. Effect of age on

673 hematopoiesis in man. Blood 63, 502-509 (1984).

674 2. de Haan, G. \& Lazare, S. S. Aging of hematopoietic stem cells. Blood 131, 479-487

$675 \quad$ (2018).

676 3. Mohrin, M. et al. Hematopoietic stem cell quiescence promotes error-prone DNA repair

677 and mutagenesis. Cell Stem Cell 7, 174-185 (2010).

678 4. Jaiswal, S. \& Ebert, B. L. Clonal hematopoiesis in human aging and disease. Science $679 \quad 366,(2019)$.

680 5. Jaiswal, S. et al. Age-related clonal hematopoiesis associated with adverse outcomes. N. Engl. J. Med. 371, 2488-2498 (2014).

682

6. Genovese, G. et al. Clonal hematopoiesis and blood-cancer risk inferred from blood DNA sequence. N. Engl. J. Med. 371, 2477-2487 (2014).

7. Xie, M. et al. Age-related mutations associated with clonal hematopoietic expansion and malignancies. Nat. Med. 20, 1472-1478 (2014).

8. McKerrell, T. et al. Leukemia-associated somatic mutations drive distinct patterns of age-related clonal hemopoiesis. Cell Rep. 10, 1239-1245 (2015).

9. Abelson, S. et al. Prediction of acute myeloid leukaemia risk in healthy individuals. Nature 559, 400-404 (2018).

10. Desai, P. et al. Somatic mutations precede acute myeloid leukemia years before diagnosis. Nat. Med. 24, 1015-1023 (2018).

11. Young, A. L., Challen, G. A., Birmann, B. M. \& Druley, T. E. Clonal haematopoiesis harbouring AML-associated mutations is ubiquitous in healthy adults. Nat. Commun. $\mathbf{7}$, 12484 (2016).

12. Watson, C. J. et al. The evolutionary dynamics and fitness landscape of clonal hematopoiesis. Science (2020) doi:10.1126/science.aay9333. 
13. McKerrell, T. et al. JAK2 V617F hematopoietic clones are present several years prior to MPN diagnosis and follow different expansion kinetics. Blood Adv 1, 968-971 (2017).

14. Heuser, M. et al. Genetic characterization of acquired aplastic anemia by targeted sequencing. Haematologica 99, e165-7 (2014).

701

15. Kulasekararaj, A. G. et al. Somatic mutations identify a subgroup of aplastic anemia patients who progress to myelodysplastic syndrome. Blood 124, 2698-2704 (2014).

16. Lane, A. A. et al. Low frequency clonal mutations recoverable by deep sequencing in patients with aplastic anemia. Leukemia 27, 968-971 (2013).

705

17. Yoshizato, T. et al. Somatic Mutations and Clonal Hematopoiesis in Aplastic Anemia. N. Engl. J. Med. 373, 35-47 (2015).

18. Coombs, C. C. et al. Therapy-Related Clonal Hematopoiesis in Patients with Nonhematologic Cancers Is Common and Associated with Adverse Clinical Outcomes. Cell Stem Cell 21, 374-382.e4 (2017).

19. Gibson, C. J. et al. Clonal Hematopoiesis Associated With Adverse Outcomes After Autologous Stem-Cell Transplantation for Lymphoma. J. Clin. Oncol. 35, 1598-1605 (2017).

20. Wong, T. N. et al. Role of TP53 mutations in the origin and evolution of therapy-related acute myeloid leukaemia. Nature 518, 552-555 (2015).

21. Meisel, M. et al. Microbial signals drive pre-leukaemic myeloproliferation in a Tet2-

22. Bick, A. G. et al. Inherited causes of clonal haematopoiesis in 97,691 whole genomes. Nature 586, 763-768 (2020).

719 23. Hinds, D. A. et al. Germ line variants predispose to both JAK2 V617F clonal hematopoiesis and myeloproliferative neoplasms. Blood 128, 1121-1128 (2016).

721 24. Zink, F. et al. Clonal hematopoiesis, with and without candidate driver mutations, is common in the elderly. Blood 130, 742-752 (2017). 
723

724

725

726

727

728

729

730

731

732

733

734

735

736

737

738

739

740

741

742

743

744

745

746

747

748

25. Orrù, V. et al. Genetic variants regulating immune cell levels in health and disease. Cell 155, 242-256 (2013).

26. Martincorena, I. et al. Universal Patterns of Selection in Cancer and Somatic Tissues. Cell 171, 1029-1041.e21 (2017).

27. Beerenwinkel, N. et al. Genetic progression and the waiting time to cancer. PLoS Comput. Biol. 3, e225 (2007).

28. Blokzijl, F. et al. Tissue-specific mutation accumulation in human adult stem cells during life. Nature 538, 260-264 (2016).

29. Lee-Six, H. et al. Population dynamics of normal human blood inferred from somatic mutations. Nature 561, 473-478 (2018).

30. Baron, C. S. \& van Oudenaarden, A. Unravelling cellular relationships during development and regeneration using genetic lineage tracing. Nat. Rev. Mol. Cell Biol. 20, 753-765 (2019).

31. Abascal, F. et al. Somatic mutation landscapes at single-molecule resolution. Nature 593, 405-410 (2021).

32. Mitchell, E. et al. Clonal dynamics of normal haematopoiesis with human ageing. (submitted as companion manuscript) (2021).

33. Karcher, M. D., Palacios, J. A., Lan, S. \& Minin, V. N. phylodyn: an R package for phylodynamic simulation and inference. Mol. Ecol. Resour. 17, 96-100 (2017).

34. Lan, S., Palacios, J. A., Karcher, M., Minin, V. N. \& Shahbaba, B. An efficient Bayesian inference framework for coalescent-based nonparametric phylodynamics.

$$
\text { Bioinformatics 31, 3282-3289 (2015). }
$$

35. Osorio, F. G., Huber, A. R., Oka, R., Verheul, M. \& Patel, S. H. Somatic mutations reveal lineage relationships and age-related mutagenesis in human hematopoiesis. Cell Rep. (2018).

36. Desai, P. et al. Somatic mutations precede acute myeloid leukemia years before 
diagnosis. Nature Medicine vol. 24 1015-1023 (2018).

37. Papaemmanuil, E. et al. Genomic Classification and Prognosis in Acute Myeloid Leukemia. N. Engl. J. Med. 374, 2209-2221 (2016).

38. Papaemmanuil, E. et al. Clinical and biological implications of driver mutations in myelodysplastic syndromes. Blood 122, 3616-27; quiz 3699 (2013).

39. Martincorena, I. \& Campbell, P. J. Somatic mutation in cancer and normal cells. Science 349, 1483-1489 (2015).

756

40. Haferlach, T. et al. Landscape of genetic lesions in 944 patients with myelodysplastic syndromes. Leukemia 28, 241-247 (2014).

758

41. Schwartz, J. R. et al. The genomic landscape of pediatric myelodysplastic syndromes. Nat. Commun. 8, 1557 (2017).

42. Takita, J. et al. Novel splicing-factor mutations in juvenile myelomonocytic leukemia. Leukemia 26, 1879-1881 (2012).

43. Latchney, S. E. \& Calvi, L. M. The aging hematopoietic stem cell niche: Phenotypic and functional changes and mechanisms that contribute to hematopoietic aging. Semin.

44. Griffith, J. F. Age-Related Changes in the Bone Marrow. Current Radiology Reports 5, 24 (2017).

45. Fabre, M. A. et al. Concordance for clonal hematopoiesis is limited in elderly twins. Blood 135, 269-273 (2020).

46. Gerstung, M., Papaemmanuil, E. \& Campbell, P. J. Subclonal variant calling with multiple samples and prior knowledge. Bioinformatics vol. 30 1198-1204 (2014).

47. Jones, D. et al. cgpCaVEManWrapper: Simple Execution of CaVEMan in Order to

772 Detect Somatic Single Nucleotide Variants in NGS Data. Curr. Protoc. Bioinformatics 56, 15.10.1-15.10.18 (2016).

774 48. Raine, K. M. et al. cgpPindel: Identifying Somatically Acquired Insertion and Deletion 

(2015).

49. Miles, L. A. et al. Single-cell mutation analysis of clonal evolution in myeloid malignancies. Nature 587, 477-482 (2020).

50. Golding, N. greta: simple and scalable statistical modelling in R. Journal of Open Source Software 4, 1601 (2019).

781

51. Beerenwinkel, N., Schwarz, R. F., Gerstung, M. \& Markowetz, F. Cancer evolution: mathematical models and computational inference. Syst. Biol. 64, e1-25 (2015).

783

52. Ellis, P. et al. Reliable detection of somatic mutations in solid tissues by laser-capture microdissection and low-input DNA sequencing. Nat. Protoc. 16, 841-871 (2021).

785

53. Moore, L. et al. The mutational landscape of normal human endometrial epithelium.

786 Nature 580, 640-646 (2020).

54. Van Loo, P. et al. Allele-specific copy number analysis of tumors. Proc. Natl. Acad. Sci. U. S. A. 107, 16910-16915 (2010).

55. Spencer Chapman, M. et al. Lineage tracing of human development through somatic mutations. Nature 595, 85-90 (2021).

56. Hoang, D. T. et al. MPBoot: fast phylogenetic maximum parsimony tree inference and bootstrap approximation. BMC Evol. Biol. 18, 11 (2018).

793 57. R Core Team. R: A Language and Environment for Statistical Computing. (2020). 


\section{Extended data figures}

a

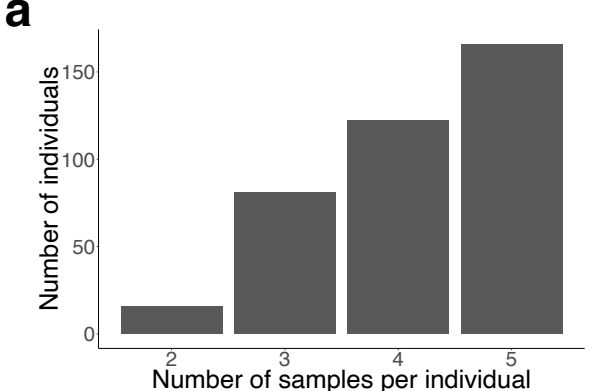

b

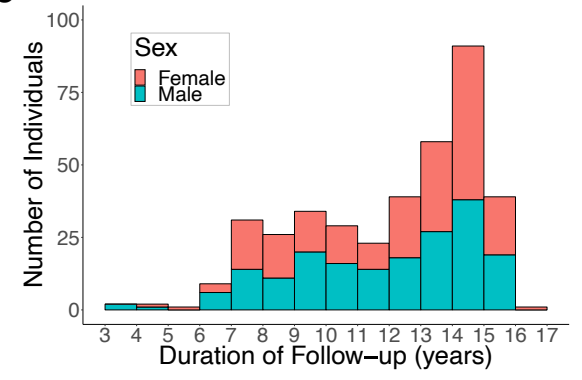

C

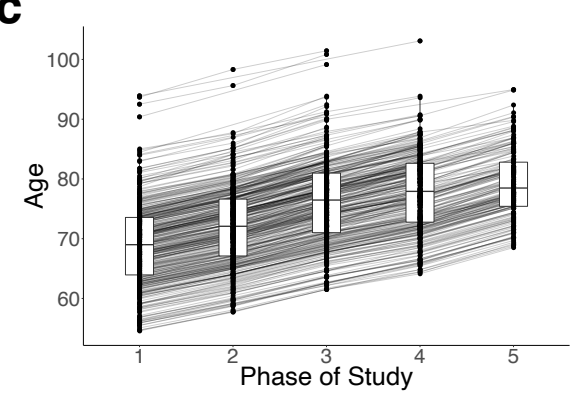

796 Extended Data Fig. 1: Longitudinal cohort characteristics. a, Distribution of the number of serial samples obtained per individual. b, Duration of follow-up per individual. c,

798 Distribution of participants' ages at each of the five sampling phases of the SardiNIA study. 


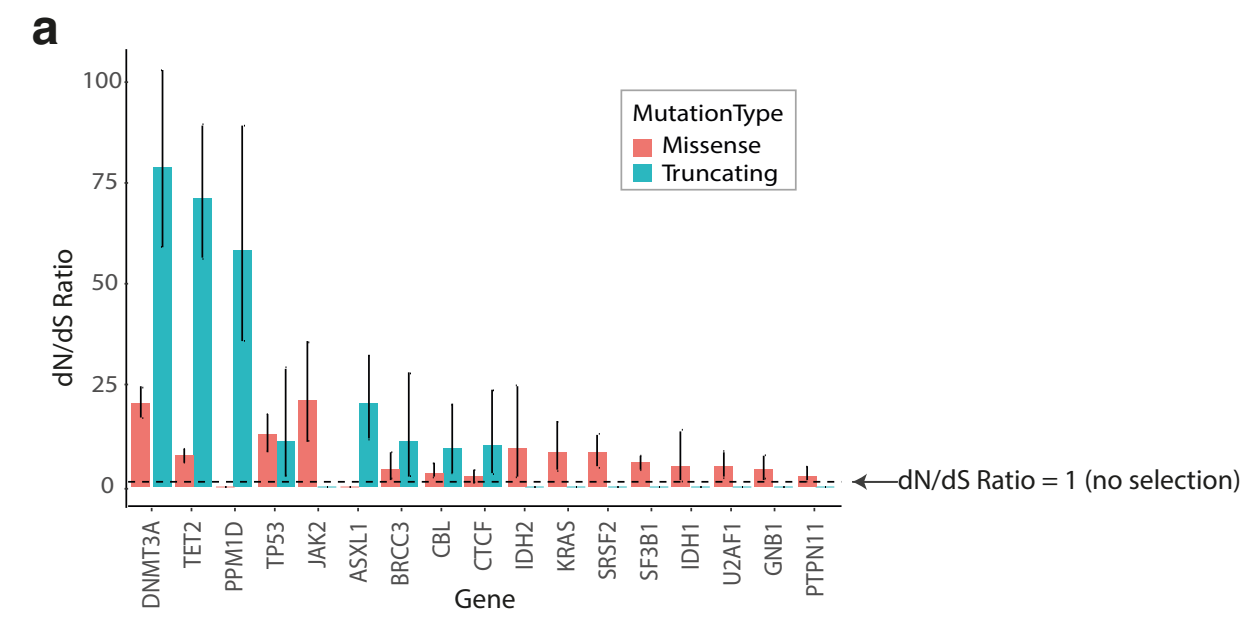

b

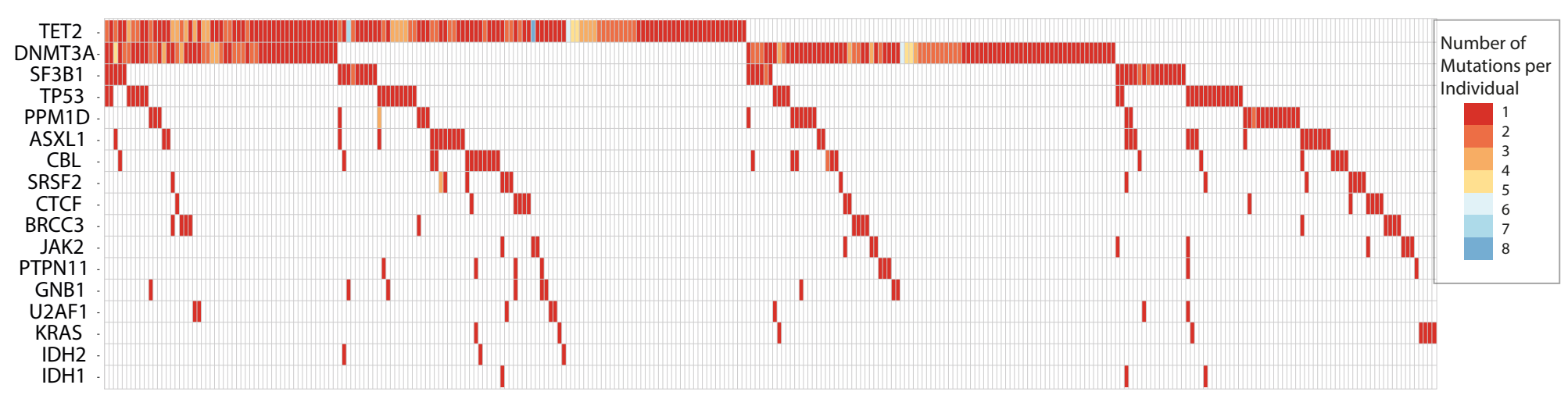

Extended Data Fig. 2: Mutation prevalence and selection in different genes. a, Observed-to-expected (dN/dS) ratios for the 17 genes with missense and/or truncating mutations under positive selection (with $\mathrm{q}<0.1$ ). The dashed line indicates a dN/dS value of 1 , which represents neutrality (no selection). $\mathbf{b}$, Waterfall plot showing the number and distribution of mutations among participants. Each column represents 1 individual, and each row 1 gene. Coloured squares indicate the presence of a mutation with the specific colour indicating the number of distinct mutations in that gene identified in that individual. For individuals with the same mutation identified at multiple serial timepoints, the serially-observed mutation is counted only once. 
bioRxiv preprint doi: https://doi.org/10.1101/2021.08 12.455048; this version posted August 12 2021. The copyright holder for this preprint

(which was not certified by peer review) is the author/funder, who has granted bioRxiv a licepse to display the prepkint in perpetuity. If is

TET2

made available under aCC-BY-NC-ND 4.0 Internatiổnal ficense.

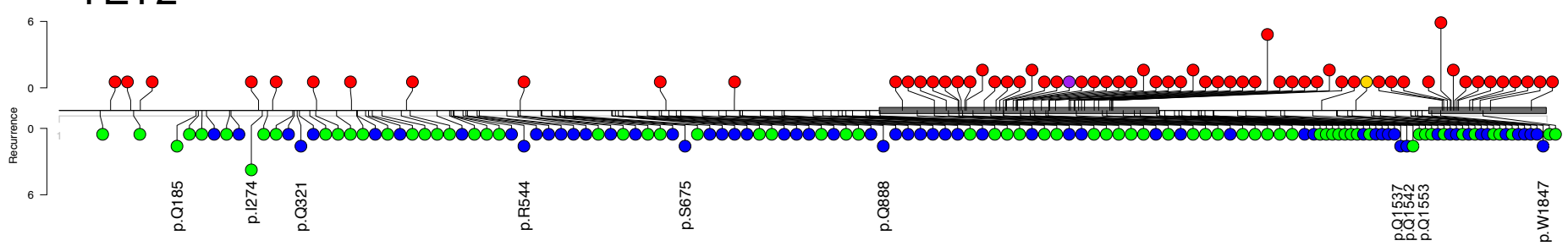

DNMT3A

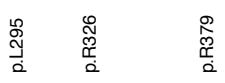

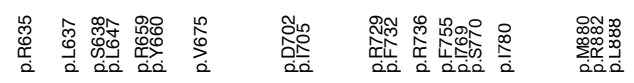
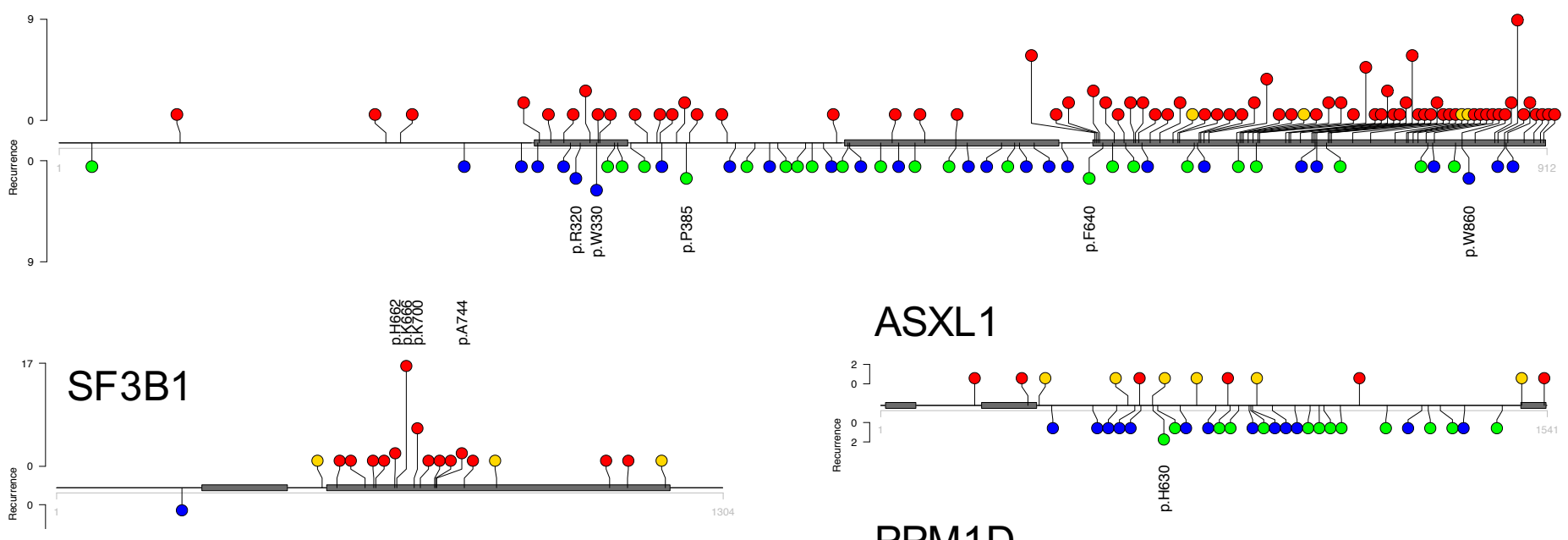

ASXL1
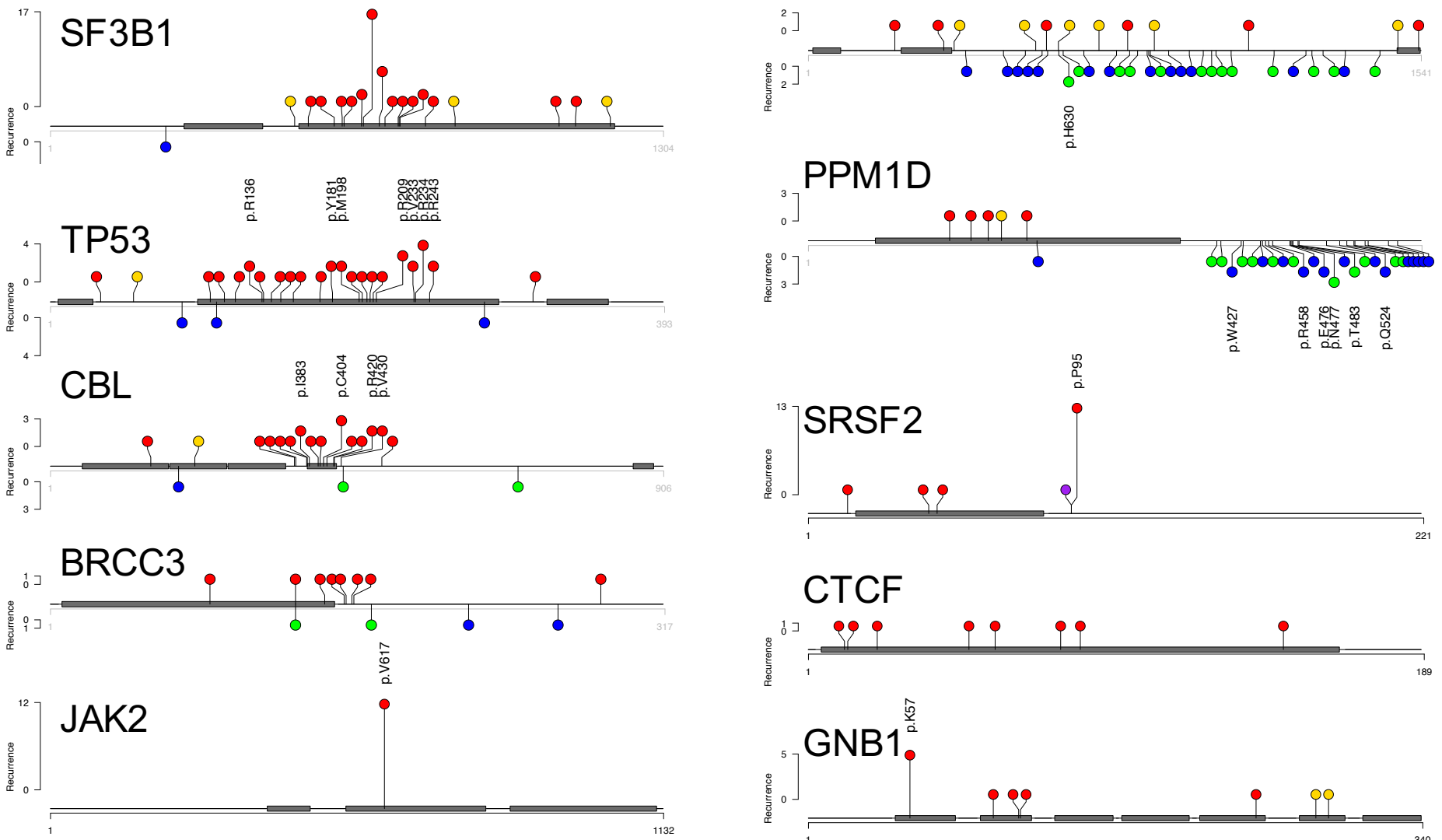

\section{CTCF}

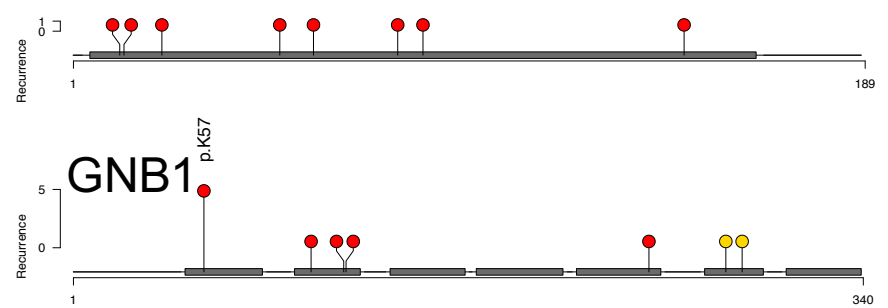

\section{PTPN11}
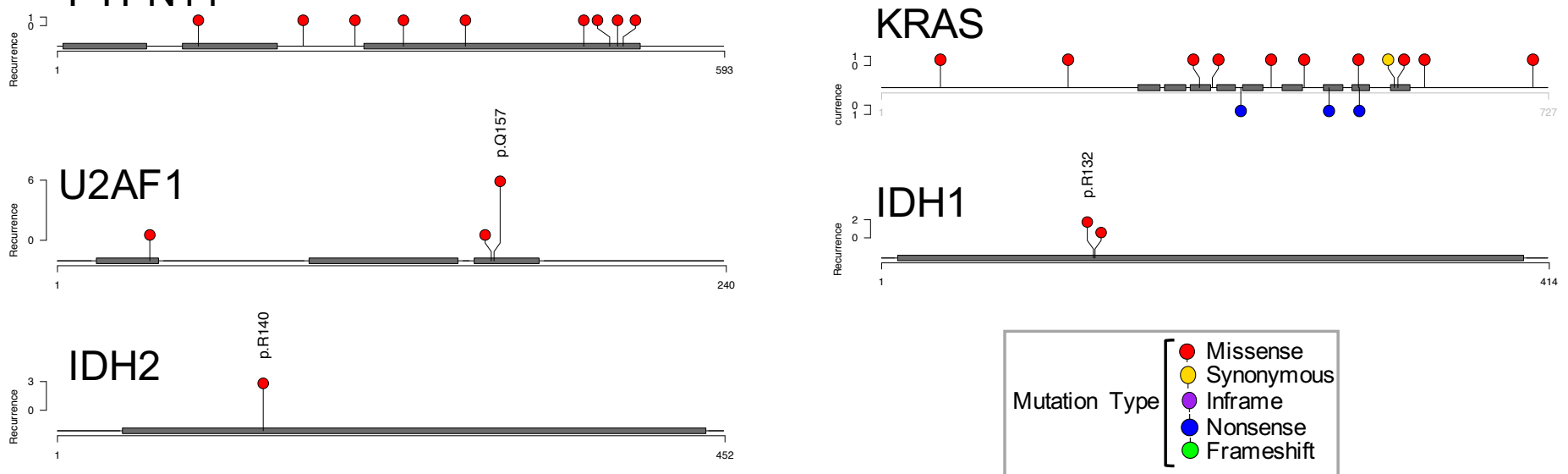
bioRxiv preprint doi: https://doi.org/10.1101/2021.08.12.455048; this version posted August 12, 2021. The copyright holder for this preprint (which was not certified by peer review) is the author/funder, who has granted bioRxiv a license to display the preprint in perpetuity. It is

Extended Data Fig. 3: Distribution of somatic mutations within driver genes (previous page). Lolliplots show the longest protein isoform of each gene, with protein domains depicted by grey rectangles. Each circle represents a somatic mutation. The vertical distance of the circle from the protein cartoon indicates its recurrence in the cohort (quantified on the $y$-axis). Amino acid codons recurrently mutated (ie. observed in more than one individual) in our cohort are explicitly labelled. Circle colours indicate the mutation type as per key. Non-truncating mutations (missense, inframe, synonymous) are depicted above and truncating mutations (nonsense, frameshift) below the protein cartoon. 
bioRxiv preprint doi: https://doi.org/10.1101/2021.08.12.455048; this version posted August 12, 2021. The copyright holder for this preprint

(which was not certified by peer review) is the author/funder, who has granted bioRxiv a license to display the preprint in perpetuity. It is made available under aCC-BY-NC-ND 4.0 International license.
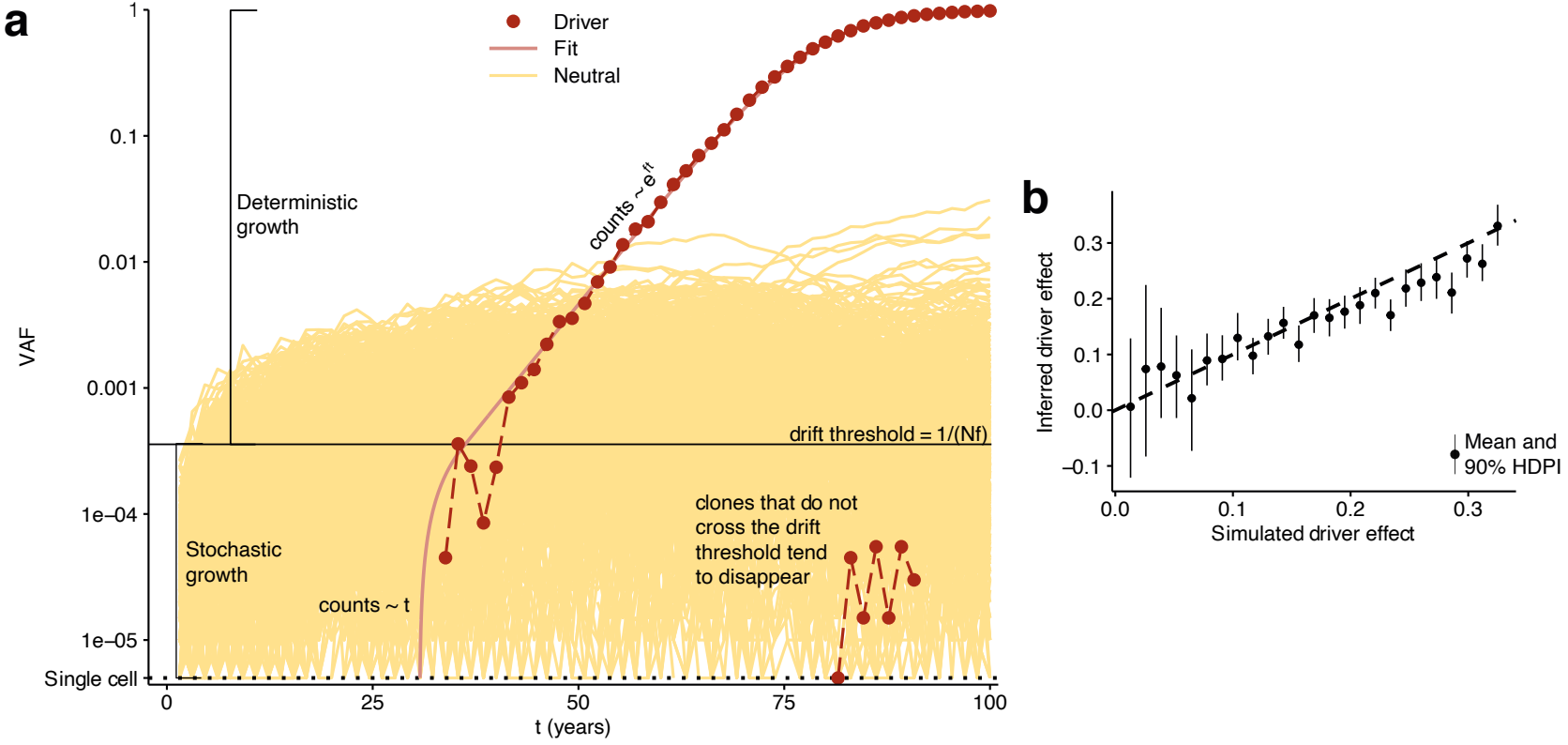

C Inferred (dark red) and observed (golden) trajectories for recurrent sites
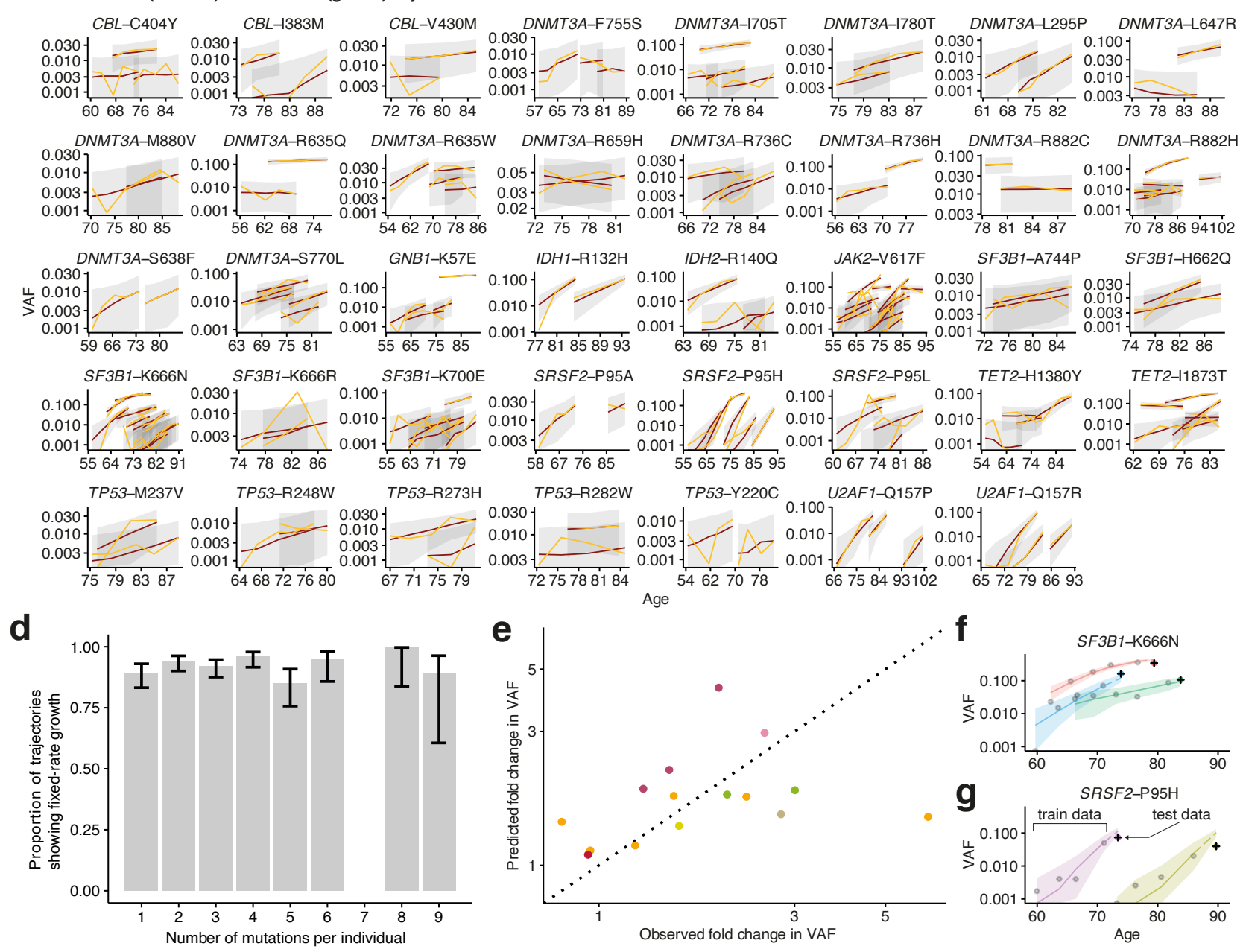

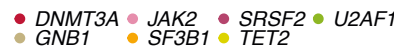

h

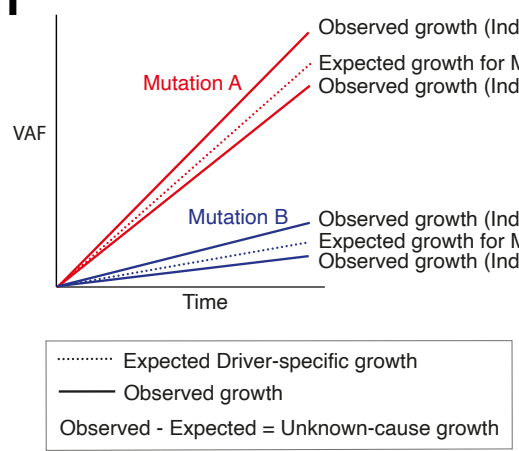

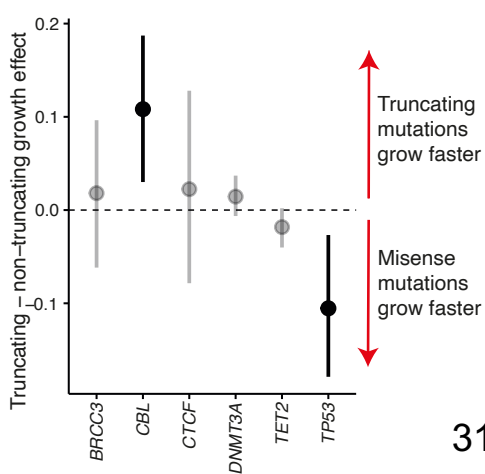


bioRxiv preprint doi: https://doi.org/10.1101/2021.08.12.455048; this version posted August 12, 2021. The copyright holder for this preprint (which was not certified by peer review) is the author/funder, who has granted bioRxiv a license to display the preprint in perpetuity. It is

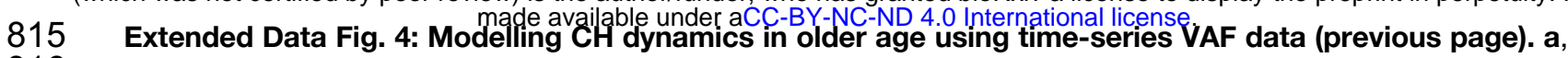

816 Representation of a Wright-Fisher simulation, showing two phases of clonal growth. The likelihood of a clone

817 transitioning from stochastic to deterministic growth is inversely proportional to the product of its fitness (f) and

818 the total number of stem cells $(\mathrm{N})$. Clones with no fitness advantage (depicted in yellow) are unlikely to exceed

819 their drift thresholds and tend to disappear or remain undetectable. Fitter clones (depicted in red) are more likely

820 to reach deterministic growth. $\mathbf{b}$, Association between the driver mutation effect used in the Wright-Fisher

821 simulations and the driver effect inferred using our model $\left(R^{2}=0.92\right)$. c, Comparison of observed (golden) and

822 inferred (red) trajectories for all recurrently mutated sites. Grey bands represent $95 \%$ highest posterior density intervals. d, Relationship between the number of mutations co-occurring within an individual and the proportion of clones growing at a fixed rate over time. e, Association between VAF predicted by our model, and VAF observed in additional prospectively-collected samples from 11 individuals with $15 \mathrm{CH}$ driver mutations, not used to infer clonal growth rate in our model. The dotted line is along the diagonal, depicting theoretical perfect agreement between predicted and observed VAF. f,g, Example trajectories of clones with SF3B1-K666N (f) and SRSF2-P95H (g) mutations. Points represent VAFs used in our model to fit the growth curve (train), and crosses represent prospectively tested VAFs used (test), showing good agreement between predicted and observed VAFs. $\mathbf{h}$, Illustration of the determinants of growth in our model. Each mutant gene and/or site drives an expected rate of clonal growth. In this example, Mutation A is expected to drive faster growth than Mutation B. The growth rates of different clones bearing the same mutation, either in different individuals or in distinct clones within the same individual, can differ. Some grow faster than expected from the identity of the driver mutation (eg. Individual 1 with Mutation A), and some grow slower (eg. Individual 2 with Mutation A). The residual term in our model, the difference between observed and expected growth rate, is referred to as "unknown-cause growth". $\mathbf{i}$, Comparison of growth rate associated with truncating vs non-truncating mutations in genes with both driver types. Points above the dashed line show faster growth of truncating mutations, and points below show faster non-truncating mutations. 
bioRxiv preprint doi: https://doi.org/10.1101/2021.08.12.455048; this version posted August 12, 2021. The copyright holder for this preprint (which was not certified by peer review) is the author/funder, who has granted bioRxiv a license to display the preprint in perpetuity. It is made available under aCC-BY-NC-ND 4.0 International license.

a

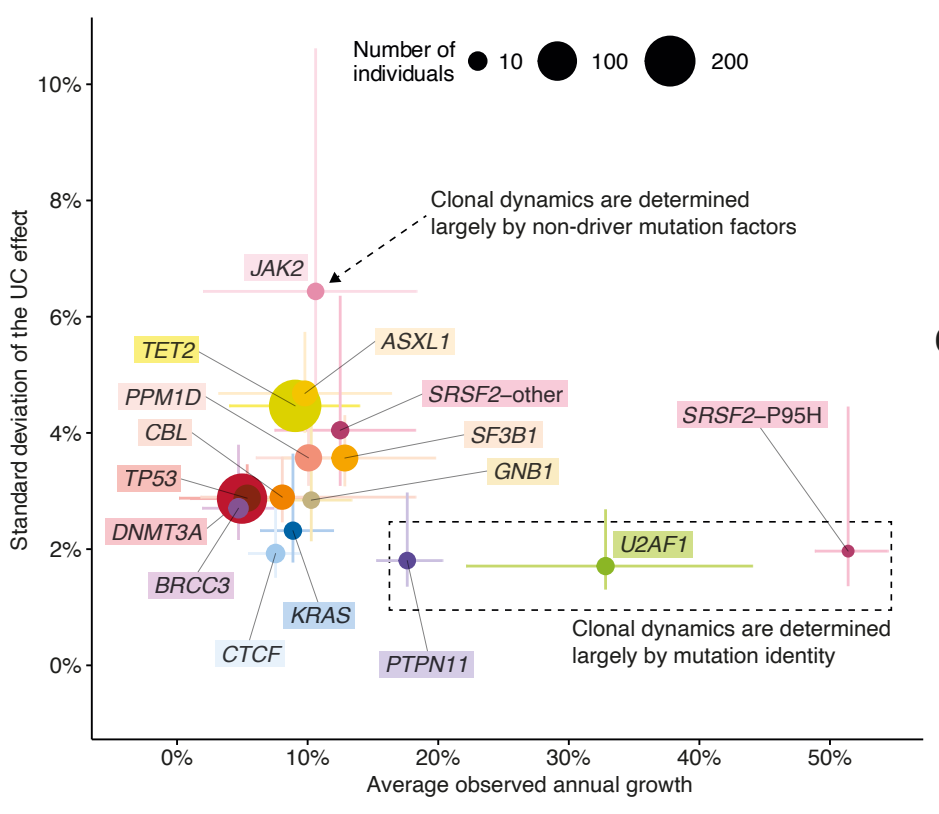

b

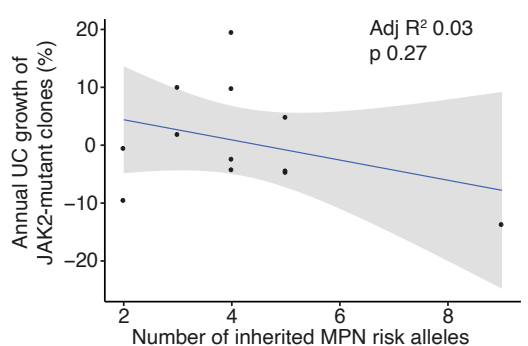

C

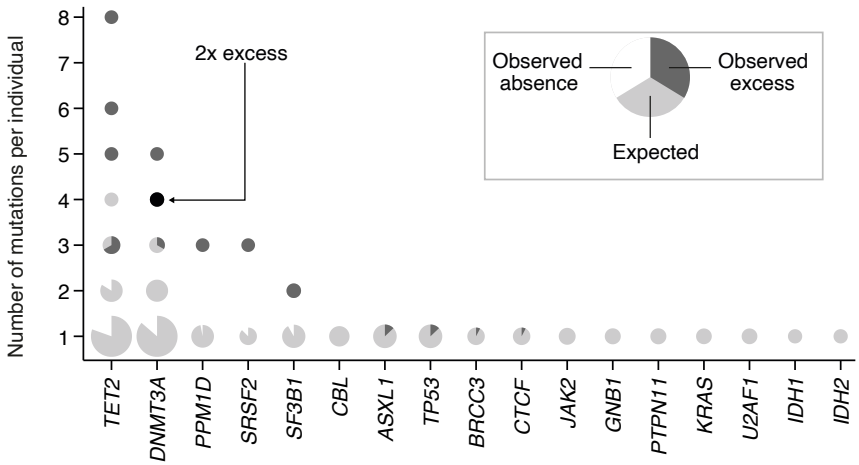

d

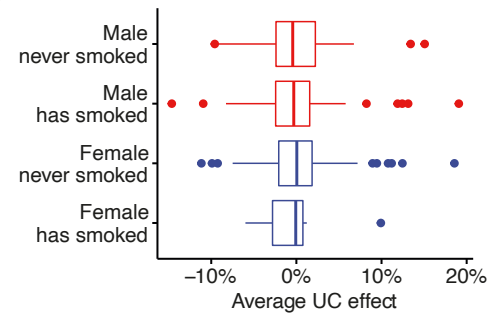

e

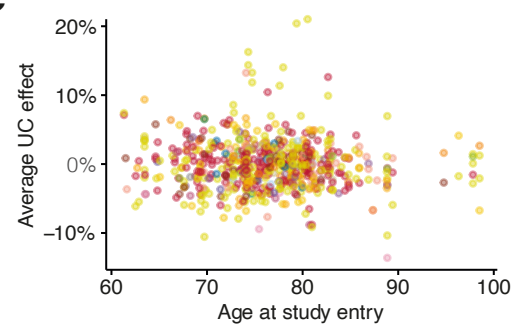

f

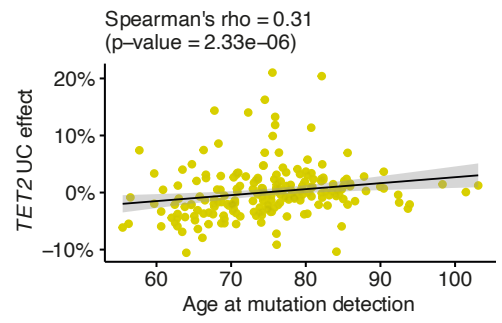

Extended Data Fig. 5: Differences in growth rate between individuals/clones with the same driver. a, For each gene, we contrast the mean annual growth rate among individuals/clones bearing a mutation in that gene, with the spread in this rate (defined here as the standard deviation of the unknown-cause (UC) growth). Circles represent point estimates, with circle size indicating the number of clones bearing a mutation in that gene, and lines representing the $90 \%$ confidence interval $(\mathrm{Cl})$. For the standard deviation, the $90 \% \mathrm{Cl}$ was calculated assuming that $\frac{(n-1) s^{2}}{\sigma^{2}} \sim \operatorname{Chis} q(n-1)$, with $n$ being the sample size, $s$ the standard deviation estimate and $\sigma^{2}$ the true population variance. SRSF2-P95H mutations are plotted separately to other SRSF2 mutations, as they are associated with significantly different growth dynamics. b. Relationship between number of inherited MPN risk alleles and JAK2-mutant clonal growth rate. c, The number of mutations per individual in each gene is plotted. Each data-point is a pie-chart, the size of which reflects the number of individuals. For each gene, given the observed mutation prevalence in our cohort, the pie is fully light grey if the number of individuals we observed with a specific number of mutations is the same as the number of individuals we expected by chance. The presence of a white segment indicates that we found fewer individuals with that number of mutations, compared to expected. The presence of a dark grey segment indicates that we found an excess of individuals with that number of mutations. We estimate the expected number of mutations in each gene in each individual through Monte Carlo estimation; assuming the prevalence of mutations in the cohort is uniform for each gene across individuals, we simulate 1,000 scenarios where we randomly distribute these mutations given the number of mutations in each individual. d, Association between sex and smoking history and the average UC effect for each individual (n.s.). e, Association between age at study entry and the average UC effect for each individual (n.s.). f, Association between age at mutation detection and UC effect for each TET2-mutant clone (Spearman's rho = $860 \quad 0.31 ; p$-value $=2.33^{*} 10^{-6}$ ). 
bioRxiv preprint doi: https://doi.org/10.1101/2021.08.12.455048; this version posted August 12, 2021. The copyright holder for this preprint (which was not certified by peer review) is the author/funder, who has granted bioRxiv a license to display the preprint in perpetuity. It is made available under aCC-BY-NC-ND 4.0 International license.

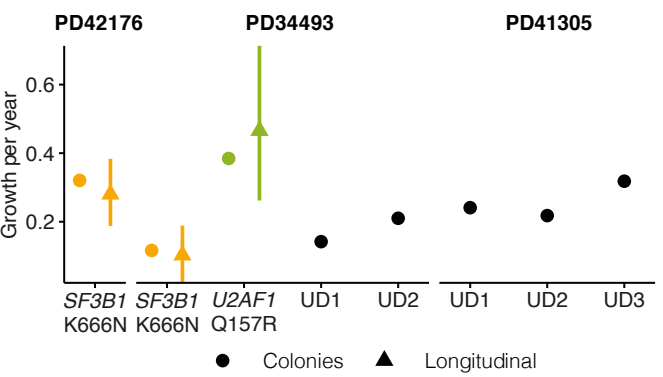

Extended Data Fig. 6: Lifelong growth in phylogenetic trees. Comparison between annual growth derived from phylogenies and growth observed in longitudinal data. For the phylogenies this was obtained by fitting an exponential growth curve to the entire phylodynamic trajectory. 
bioRxiv preprint doi: https://doi.org/10.1101/2021.08.12.455048; this version posted August 12, 2021. The copyright holder for this preprint (which was not certified by peer review) is the author/funder, who has granted bioRxiv a license to display the preprint in perpetuity. It is made available under aCC-BY-NC-ND 4.0 International license.

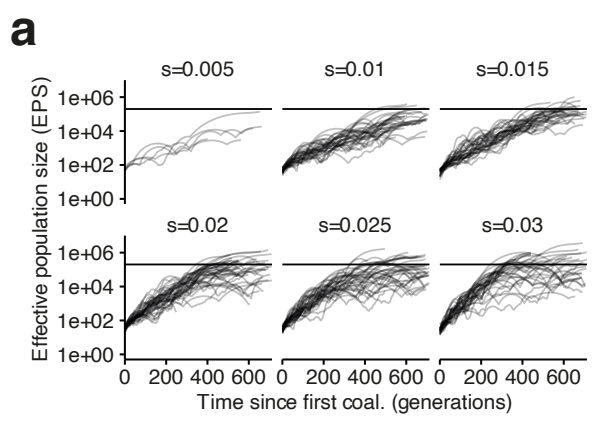

C

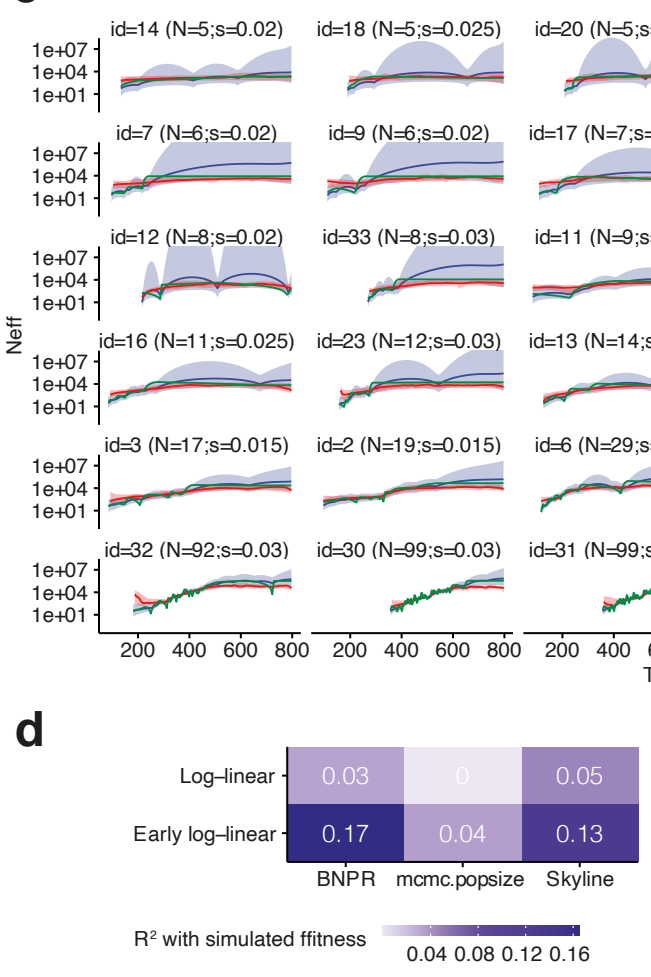

e

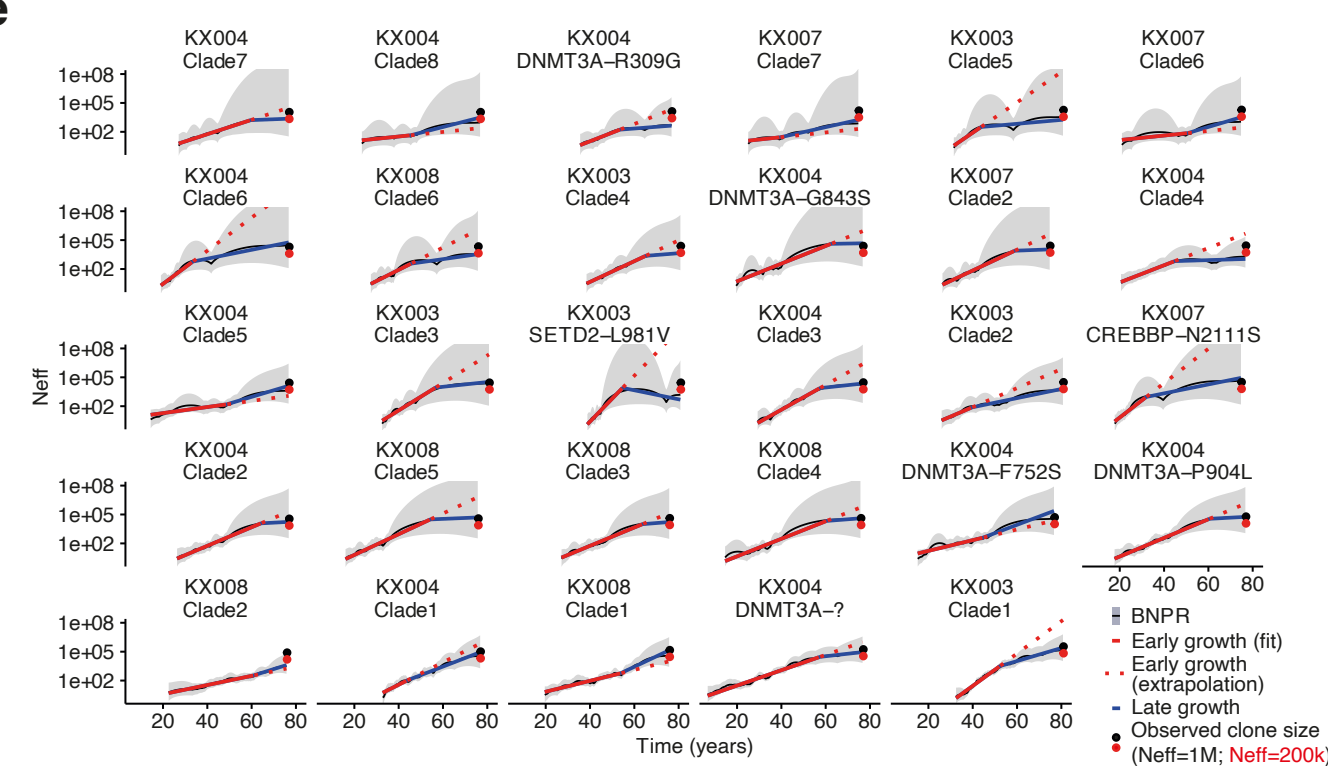

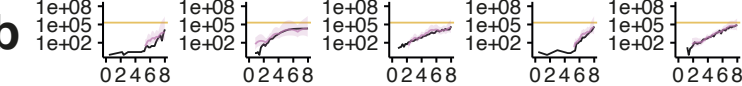

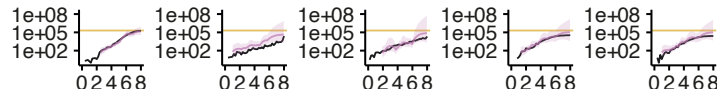

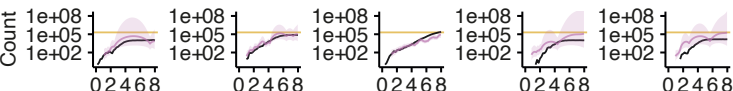

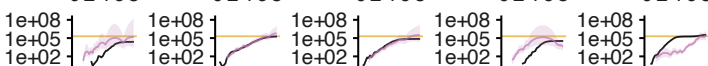

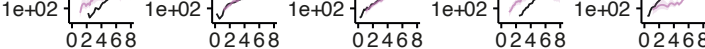

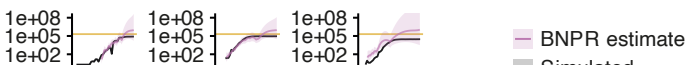
02468 - BNPR estim 
bioRxiv preprint doi: https://doi.org/10.1101/2021.08.12.455048; this version posted August 12, 2021. The copyright holder for this preprint (which was not certified by peer review) is the author/funder, who has granted bioRxiv a license to display the preprint in perpetuity. It is

872 Supplementary Methods) for their estimation across a range of clade sizes and fitness effects. d, Quantification 873 of the association between true fitness values and inferred fitness values for three distinct methods of Neff 874 estimation. e, Schematic representation of all trajectories from Mitchell et al. and how extrapolating from the initial 875 growth rate leads to the overestimation of the observed clone size (here the observed clone size is obtained by 876 scaling the proportion of tips in a clade by a total Neff of either 200,000 or 1,000,000 HSC x yr). f, Quantification 877 of the deceleration effect from real data and simulations. 
bioRxiv preprint doi: https://doi.org/10.1101/2021.08.12.455048; this version posted August 12, 2021. The copyright holder for this preprint (which was not certified by peer review) is the author/funder, who has granted bioRxiv a license to display the preprint in perpetuity. It is made available under aCC-BY-NC-ND 4.0 International license.
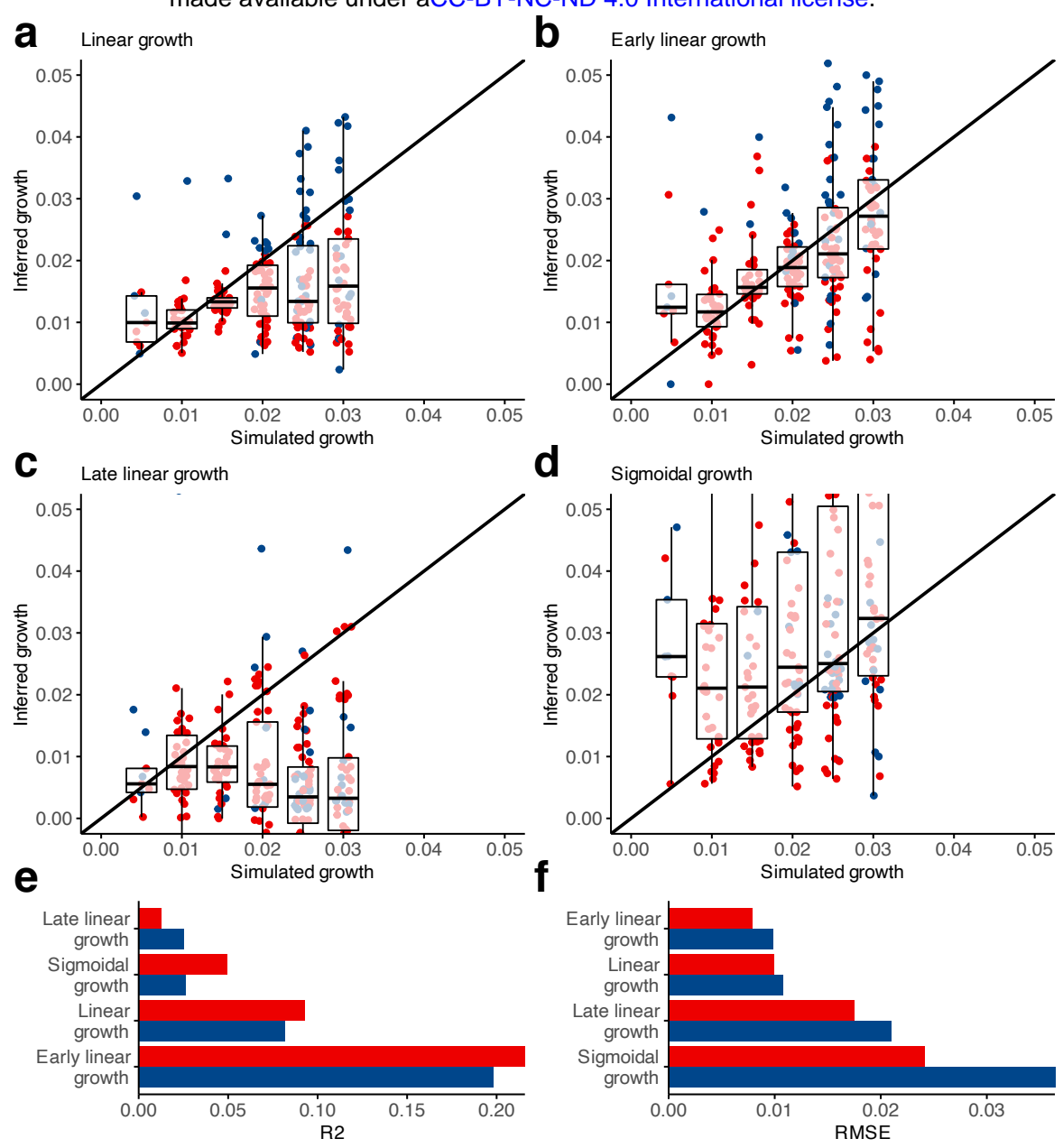

Extended Data Fig. 8: Estimation of the true clone fitness from phylodynamic estimation. Three fits were tested to estimate the true clone fitness from phylodynamic estimation of the population size and these estimates were plotted as a function of the true fitness size $(0.005,0.010,0.015,0.020,0.025$ or 0.030$)$. a, A log-linear fit; $\mathbf{b}$-c, A biphasic fit that estimates an early and a late growth rate and a change-point between both and $\mathbf{d}$, a sigmoidal fit. e, Coefficient of correlation (R2) for all four inferred coefficients. $\mathbf{f}$, Root mean squared error (RMSE) for all four inferred coefficients. In this figure red represents "low variance trajectories" (the average estimated variance for the logarithm of the trajectory is under 5) and blue represents "all trajectories". 
bioRxiv preprint doi: https://doi.org/10.1101/2021.08.12.455048; this version posted August 12, 2021. The copyright holder for this preprint (which was not certified by peer review) is the author/funder, who has granted bioRxiv a license to display the preprint in perpetuity. It is made available under aCC-BY-NC-ND 4.0 International license.
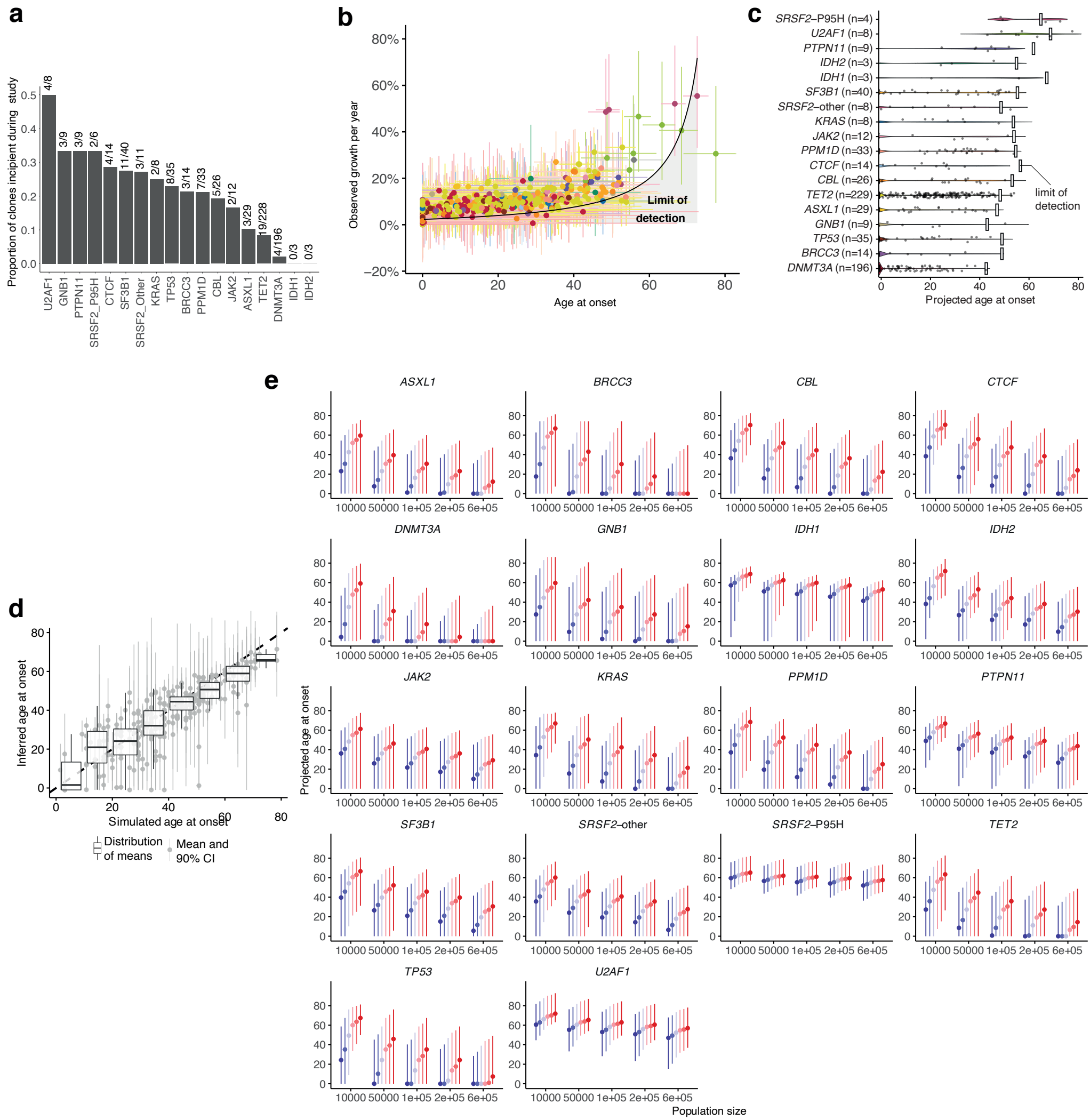

Generations/year $\begin{array}{lll}1 & 1 \\ 2 & 5 & 5\end{array}$

Extended Data Fig. 9: Age at clone detection and onset. a, Proportion of clones driven by different driver mutations that were incipient on-study, ie. undetectable at time-point 1 and detectable by the end-of-study. Absolute numbers are given above each bar. b. Relationship between age at onset and observed annual growth rate, with $90 \%$ highest posterior density intervals (HPDI). The black line and grey shaded area represent the theoretical limit of detection at 80 years of age. c, Violin plot showing the distribution of projected ages at onset for all clones, assuming stable lifelong growth at the same fixed rate we observed during older age. d, Association between the age at which clones appeared in the simulations and the age at clone foundation inferred using our time-series data $\left(R^{2}=0.75\right)$. Boxplots show that, while these estimates may have high variance, the distribution of expected values is close to the true value. e, Sensitivity analysis depicting the median (dot) and the $95 \%$ 
bioRxiv preprint doi: https://doi.org/10.1101/2021.08 12.455048; this version posted August 12, 2021. The copyright holder for this preprint

(which was not certified by peer review) is the author/funder, who has granted bioRxiv a license to display the preprint in perpetuity. It is

896 confidence interval of the ages at onset for each gene when considering different population sizes (10e3, 50e3,

$897100 \mathrm{e} 3,200 \mathrm{e} 3$ and $600 \mathrm{e} 3)$ and numbers of generations per year $(1,2,5,10,13,20)$. 
bioRxiv preprint doi: https://doi.org/10.1101/2021.08.12.455048; this version posted August 12, 2021. The copyright holder for this preprint (which was not certified by peer review) is the author/funder, who has granted bioRxiv a license to display the preprint in perpetuity. It is made available under aCC-BY-NC-ND 4.0 International license.

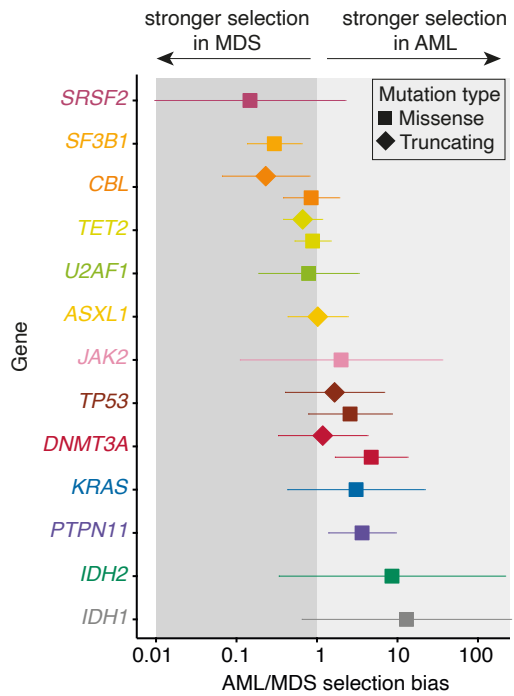

899 Extended data Fig. 10: Selection in myeloid malignancies. a, Ratio between AML dN/dS and MDS dN/dS for different genes and mutation types (missense, truncating). If this ratio is $>1$ there is a bias towards $A M L$, if it is $<1$ there is a bias towards MDS. Confidence intervals for the ratios were calculated under the assumption that $\mathrm{dN} / \mathrm{dS}$ estimates are normally distributed. 


\section{Assessing the predictive performance of clonal growth predictions}

905 Using an additional time-point (phase 6) available for 11 individuals with mutations in $C B L$ 906 (c.2434+1G>A), DNMT3A (P385fs, R882H, W330X), GNB1 (K57E), JAK2 (V617F), PPM1D 907 (Q524X), SF3B1 (K666N, K700E, R625L), SRSF2 (P95H, P95L), TET2 (Q1542X) and U2AF1 908 (Q157P, Q157R). Using the model described in the "Hierarchical modelling of clone 909 trajectories through time" section of the Methods and conditioning on the previous 910 timepoints, we predict the additional time-point and assess the predictive performance 911 through the mean absolute error (MAE) to the true VAF value.

912 Validating the dynamic coefficient and age at onset inference with Wright-Fisher 913 simulations

914 We use Wright-Fisher simulations ${ }^{1-3}$ with a fixed population of 200,000 cells and 50 possible 915 drivers, a range of fitness advantages $(0.001-0.030)$ and a range of mutation rates $(1.0 *$ $\left.91610^{-10}-4.0 * 10^{-9}\right)$. These ranges were estimated to cover the values inferred and 917 mentioned in considering that one should expect there to be approximately 13 generations of HSC per year and a population size of $200,000 \mathrm{HSC}^{4}$.

919 To simulate the conditions under which the experimental data was obtained, we fit Gamma 920 distributions to the observed coverage and observed age at first time-point truncated at the 921 minimum and maximum values for each. For each simulation we sample from these distributions the first timepoint, a random number of subsequent timepoints (between 2 and 4) from a uniform distribution and the coverage for each driver at each timepoint. We simulate the sequencing process as drawing samples from a beta-binomial distribution parameterized similarly to the one described in the "Hierarchical modelling of clone trajectories through time" section of the Methods, where the probability is the proportion of cells from a specific clone present at a given time-point. More concretely, counts $\sim B B\left(\frac{p \beta}{1-p}, \beta, c o v\right)$, where $p$ is the allele frequency of a mutation, $\beta$ is the technical overdispersion parameter and $\operatorname{cov}$ is the coverage which is sampled from the coverage distribution as inferred from our data.

930

To infer coefficients under this setting we converted generations to years (13 generations per year) and used the framework described in the previous sections to infer these coefficients. Since the nature of these mutations does not consider different levels of genetic resolution, we had to modify the driver coefficient to driver effect $\sim N\left(0, \sqrt{2 * 0.1^{2}}\right)$ so that the distribution from which this coefficient is being drawn has the one we consider for the driver effect considering a gene, domain and site effect. The observed coefficients are converted to year as coefficients $=\left(1+\right.$ fitness $^{g}-1$, where $g$ is the number of generations per year, and we assess the fit between inferred and observed coefficients considering these values. We additionally calculate the age at clone foundation for the inferred coefficients and, using these simulations which allow us to know the true age at clone foundation, we assess the fit between inferred and observed ages at clone foundation.

941 To better understand the impact that population size and generation times have on these

942 simulations, we conduct the same analysis considering two additional scenarios: a 943 population size of 100,000 HSC and 5 generations per year, and a population size of 50,000 944 HSC and 1 generation per year.

945 Finally, we also calculate the age at onset as specified in the "Determining the expected age 946 at beginning of clone onset". To do this, we assume that these clones follow a Wright-Fisher 
bioRxiv preprint doi: https://doi.org/10.1101/2021.08.12.455048; this version posted August 12,2021 . The copyright holder for this preprint (which was not certified by peer review) is the author/funder, who has granted bioRxiv a license to display the preprint in perpetuity. It is made available under aCC-BY-NC-ND 4.0 International license.

947 process, where growth can be separated into two distinct phases which depend on the size

948 of the clone - a stochastic phase, where the clone is too small and during which growth

949 happens linearly, and a deterministic phase, during which growth is approximately

950 exponential (Extended Data Fig. 4a). According to this growth regime, the age at onset can

951 be calculated as $t_{0}$ adjusted $=t_{0}+\frac{\log \left(g / b_{\text {total }}\right)}{b_{\text {total }}}-\frac{1}{b_{\text {total }}}$, where $t_{0}$ is the age at onset if the

952 clone grew exponentially (as opposed to following a Wright-Fisher process), $\frac{\log \left(g / b_{\text {total }}\right)}{b_{\text {total }}}$ is

953 the time at which the clone started to grow deterministically and $\frac{1}{b_{\text {total }}}$ is the expected time

954 the clone spends following a stochastic growth regime. We assess the validity of this

955 approach by calculating the coefficient of correlation between inferred and true ages at onset

956 from the simulations.

957 Validating annual growth rate inferences from single-cell phylogenies with 958 Wright-Fisher simulations

959 We use Wright-Fisher simulations ${ }^{2,3}$ with 50 possible drivers and test a range of different 960 fitness advantages ([0.005,0.010,0.015,0.020,0.025,0.030]) over 800 generations at a fixed 961 population size of 200,000 HSC. For each fitness effect we define a driver mutation rate $962\left(\left[200 * 10^{-9}, 50 * 10^{-9}, 20 * 10^{-9}, 15 * 10^{-9} \quad{ }^{-9}, 8 * 10^{-9} \quad{ }^{-9}, 5 * 10^{-9}\right]\right.$, respectively) that 963 guarantees that at least a few simulations lead to clones which expand to sufficient sizes and 964 avoid many competing expansions and keep the passenger mutation rate constant $(2 *$ $965{ }^{1} 1^{-5}$ ). For each simulation we infer phylogenetic trees by sampling 100 representative 966 clones from our population and using a neighbour-joining algorithm based on mutation presence. The representative sampling is done by defining for each clone a probability of being sampled that is equivalent to its proportion in the population. We then detect the clades

969 that contain drivers, isolate them and infer their effective population size (Neff) trajectory using BNPR $^{5,6}$.

We fit different models to the inferred Neff trajectories, namely:

1. A log-linear fit (assumes exponential growth);

2. A scaled and shifted sigmoidal fit (assumes that growth saturates based on the Neff trajectory);

3. A shifted sigmoidal fit (assumes that growth saturates at 1 and that the most recent Neff estimate corresponds to the proportion of tips in the clade);

4. A biphasic log-linear fit (assumes that growth is exponential and has two distinct coefficients corresponding to early and late growth; the boundary between early and late growth - otherwise referred to as the changepoint between both - is also fitted with the other parameters and is constrained to lie in the central part of the trajectory: for the time $t$ over which the clone expands, the changepoint cannot be inferior to $\min (t)+0.25 * \operatorname{range}(t)$ nor superior to $\max (t)-0.25 * \operatorname{range}(t)$, where range $(t)=$ $\max (t)-\min (t)$. This constraint prevents fits that are too close to the clonal inception or to the clone at later stages).

We compare these models by assessing how closely they are able to recapitulate the original fitness in the simulations. To do so, we calculate their coefficient of determination and root mean squared error. We also visually assess how similar these trajectories are to the true driver trajectories as reconstructed from simulations - to match clones from a Wright-Fisher 
bioRxiv preprint doi: https://doi.org/10.1101/2021.08.12.455048; this version posted August 12,2021 . The copyright holder for this preprint (which was not certified by peer review) is the author/funder, who has granted bioRxiv a license to display the preprint in perpetuity. It is made available under aCC-BY-NC-ND 4.0 International license.

990 Fisher simulation to its nearest clone in a phylogenetic tree using the Hamming distance

991 between the mutations in each clone.

992 We additionally estimate the effective population size using two other methods for validation

993 - mcmc.popsize and skyline from the ape package ${ }^{7}$ in $\mathrm{R}$. This allows us to confirm our

994 observations that stem from phylodynamic estimations and that concern, mostly, a prevalent

995 effect of clonal deceleration which is detailed in the main text and in the following section.

\section{Detecting deceleration in single-cell phylogenies and longitudinal data}

997 We infer the presence of deceleration in both single-cell phylogenies and longitudinal data.

998 To do this, we use two distinct methods: calculating the ratio between the expected and 999 observed VAF and calculating deceleration using growth rates.

1000 For the first method - calculating the ratio between expected and observed VAF - we use the 1001 value for the early growth from the changepoint log-linear fit described in "Validating annual 1002 growth rate inferences from single-cell phylogenies with Wright-Fisher simulations" and 1003 extrapolate the Neff to the age at sampling. By doing so we get the expected clone fraction 1004 if growth had not changed during the Neff trajectory. We also calculated the observed clone 1005 fraction as the fraction of tips in the clade. To get the expected clone fraction from Neff we 1006 divide Neff by the inferred population size in Lee-Six et. al $(200,000 \mathrm{HSC})^{8}$. We then calculate 1007 the ratio between the expected and observed clone size - if this ratio is close to 1 this implies 1008 little to no changes in dynamics, whereas a ratio above 1 implies deceleration and a ratio 1009 below 1 implies acceleration.

1010 For the second method - calculating deceleration using growth rates - we define two distinct 1011 quantities for both single-cell phylogenies/longitudinal data - expected/observed growth, 1012 corresponding to the growth rate of each clone during observation at old age, and 1013 early/minimal historical growth, corresponding to the growth rate of each clone at an earlier 1014 stage of clonal dynamics - and calculate the ratio between them.

1015 As such, for phylogenies we first calculate the Neff trajectory for each clade using BNPR ${ }^{33}$. 1016 Next, and using their Neff trajectory, we calculate their expected growth rate by assuming a 1017 sigmoidal growth. We additionally assume that the final Neff (Neff at sampling) estimate 1018 corresponds to the fraction of tips in the clade and we scale our data accordingly such that 10191 corresponds to the maximum Neff and the fraction of tips in the clade corresponds to Neff 1020 at sampling. Thirdly and using the changepoint log-linear fit described in "Validating annual 1021 growth rate inferences from single-cell phylogenies with Wright-Fisher simulations" we derive 1022 the value for early growth. Finally, as a measure of deceleration, we calculate the ratio 1023 between expected and early growth - a value close to 1 for this ratio implies an absence of 1024 deceleration whereas smaller values imply deceleration.

1025 For the longitudinal data we use the observed growth for each clone as described in 1026 "Hierarchical modelling of clone trajectories through time". Next, we calculate the (minimal) 1027 historical growth as the growth that excludes all posterior samples that would lead to age at 1028 onset estimates exceeding lifetime (ages at onset for clones below -1, a heuristic value 1029 chosen to represent developmental onset of clones). Finally and as a measure of 1030 deceleration, we calculate the ratio between observed and historical growth. The 1031 interpretation for this ratio is similar to that defined in the previous paragraph for phylogenetic 1032 data - a value of 1 implies an absence of detectable deceleration, whereas smaller values 1033 represent the minimal amount of deceleration. This method has, however a caveat - due to 1034 the nature of this calculation (excluding posterior samples which are too slow to provide 1035 solutions within lifetime), values above 1 (indicating acceleration) are technically impossible. 


\section{Supplementary Note 1 - Determining the effect of repeated sampling 1038 on the theoretical limit of detection}

1039 Across this work we sequence individuals a median of three times across their lifetime. We

1040 define a detection threshold of $0.5 \%$ VAF as the minimum clone size for detection on

1041 individual timepoints, but the repeated sampling leads to $0.5 \%$ VAF being an

1042 overestimation of the actual limit of detection (LOD) - the size at which clones become

1043 detectable.

1044 To show this, we simulate the repeated sampling of variants existing at a true clone

1045 proportion between 0 and $2 \%$. We use this proportion $p$ as the probability parameter in a

1046 beta binomial distribution, the overdispersion $\beta$ calculated using technical replicates as the

1047 overdispersion in the same beta binomial distribution and a coverage of 1000 . Having fully

1048 parameterized this distribution (counts $\sim B B\left(\frac{p \beta}{1-p}, \beta, 1000\right.$ )) we sample counts from it

1049 between 1 to 5 times. For each combination of clone size and number of samples we

1050 perform 1,000 realisations and calculate the number of detected clones at a threshold of

$10510.5 \%$. This allows us to assess the fraction of clones with a specific size which are detected

1052 if we sample them multiple times - in other words, are able to assess the detection rate for

1053 different clone sizes and different numbers of samples.

1054 With this, we show that, at a threshold of $0.5 \%$ and sampling only once, we detect $14.8 \%$ of all clones existing at $0.5 \%$ (Supplementary Notes Fig. 1). However, repeating this sampling 3 and 5 times leads to the detection of approximately $37.7 \%$ and $54.3 \%$ of all clones existing at $0.5 \%$, respectively. As such, under regular conditions - a single sample we would detect $13.5 \%$ of all clones present at $0.5 \%$ with a detection threshold of $0.5 \%$. The question we should now ask is: what is the smallest possible clone size we detect at the same rate of detection $-13.5 \%$ - if we increase the number of samples? Using the same set of simulations, we can calculate the likely minimal size of the detected clones, summarised in Supplementary Notes Table 1, with clones as small as $0.21 \%$ and $0.14 \%$ being detected with 3 and 5 samples, respectively, using the same detection rate. As such, when considering the theoretical LOD used in Figure $4 \mathrm{k}$, we avoided using $0.5 \%$ which, as we show, would be at least twice as high as the theoretical LOD obtained from simulations. 


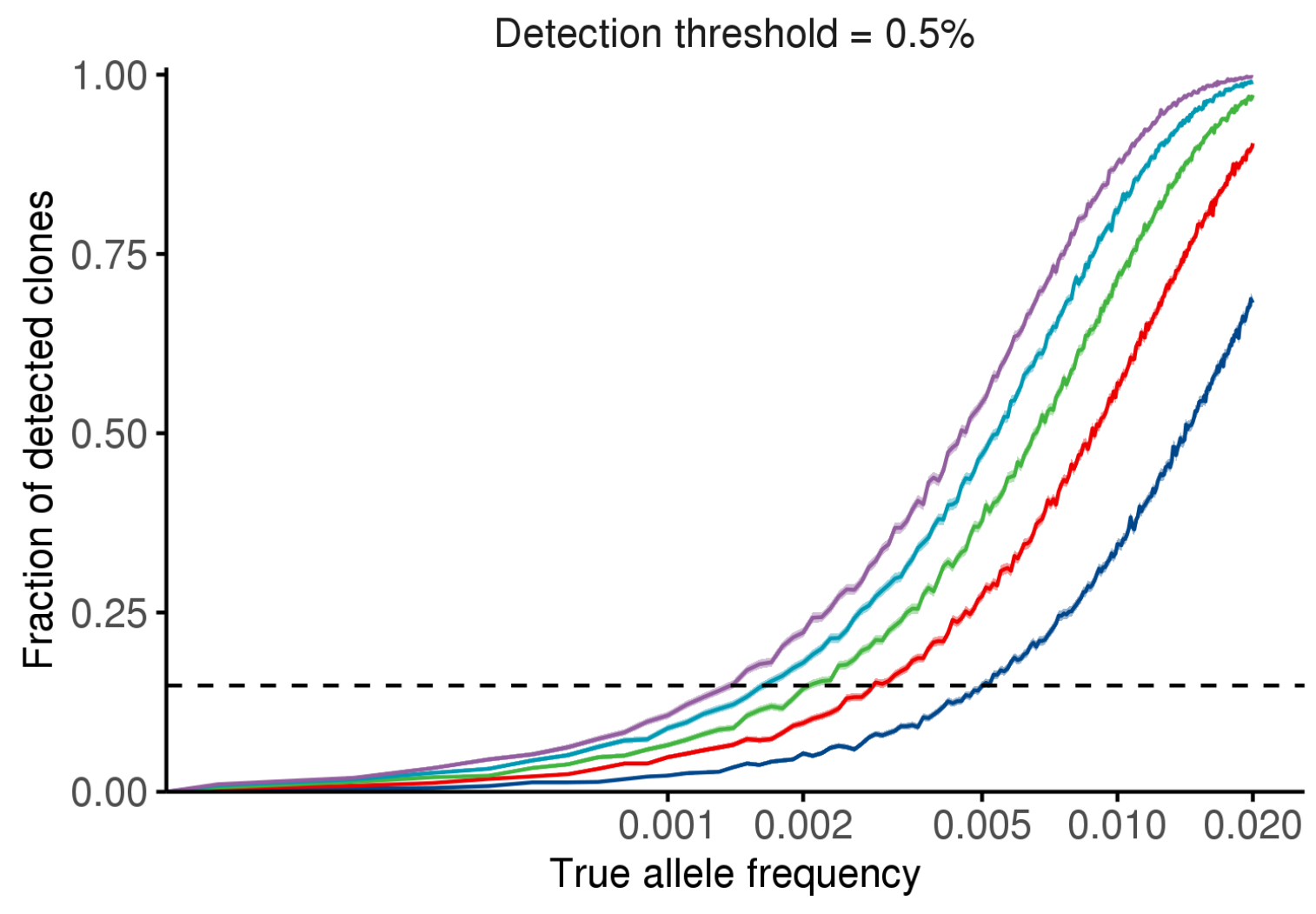

Supplementary Notes Fig. 1 - Fraction of detected clones upon repeated samples/timepoints at a detection threshold of $0.5 \%$.

Supplementary Notes Table 1 - The minimal size of detected clones using a $0.5 \%$ threshold and assuming that we are interested in detecting the same fraction of clones we would detect with a single sample at a detection threshold of $0.5 \%$.

\begin{tabular}{|l|l|}
\hline Number of samples & Minimal size of detected clones at $15.08 \%$ \\
\hline 1 & $0.50 \%$ \\
\hline 2 & $0.30 \%$ \\
\hline 3 & $0.21 \%$ \\
\hline 4 & $0.16 \%$ \\
\hline 5 & $0.14 \%$ \\
\hline
\end{tabular}

1072

\section{Supplementary references}

1. Gillespie, John H. Population Genetics: a Concise Guide. Baltimore, Md: The Johns Hopkins University Press (1998).

2. Beerenwinkel, N. et al. Genetic progression and the waiting time to cancer. PLoS Comput. Biol. 3, e225 (2007).

3. Beerenwinkel, N. \& Gerstung, M. clonex. (Github). https://github.com/gerstunglab/clonex

4. Watson, C. J. et al. The evolutionary dynamics and fitness landscape of clonal hematopoiesis. Science (2020) doi:10.1126/science.aay9333. 
5. Karcher, M. D., Malacios, J. A., Lan, S. \& Minin, V. N. phylodyn: an R package for phylodynamic simulation and inference. Mol. Ecol. Resour. 17, 96-100 (2017).

6. Lan, S., Palacios, J. A., Karcher, M., Minin, V. N. \& Shahbaba, B. An efficient Bayesian inference framework for coalescent-based nonparametric phylodynamics. Bioinformatics 31, 3282-3289 (2015).

7. Paradis, E. \& Schliep, K. ape 5.0: an environment for modern phylogenetics and evolutionary analyses in R. Bioinformatics 35, 526-528 (2019).

8. Lee-Six, H. et al. Population dynamics of normal human blood inferred from somatic mutations. Nature 561, 473-478 (2018). 